\title{
On the Tractability of Optimization Problems on $\mathrm{H}$-Graphs
}

\author{
Fedor V. Fomin ${ }^{1} \cdot$ Petr A. Golovach ${ }^{1} \cdot$ Jean-Florent Raymond ${ }^{2}$ (D) \\ Received: 10 September 2018 / Accepted: 23 February 2020 / Published online: 11 March 2020 \\ (c) The Author(s) 2020
}

\begin{abstract}
For a graph $H$, a graph $G$ is an $H$-graph if it is an intersection graph of connected subgraphs of some subdivision of $H$. $H$-graphs naturally generalize several important graph classes like interval graphs or circular-arc graph. This class was introduced in the early 1990s by Bíró, Hujter, and Tuza. Recently, Chaplick et al. initiated the algorithmic study of $\mathrm{H}$-graphs by showing that a number of fundamental optimization problems like Maximum Clique, Maximum Independent Set, or MiniMUM Dominating SET are solvable in polynomial time on $H$-graphs. We extend and complement these algorithmic findings in several directions. First we show that for every fixed $H$, the class of $H$-graphs is of logarithmically-bounded boolean-width (via mim-width). Pipelined with the plethora of known algorithms on graphs of bounded boolean-width, this describes a large class of problems solvable in polynomial time on $H$-graphs. We also observe that $H$-graphs are graphs with polynomially many minimal separators. Combined with the work of Fomin, Todinca and Villanger on algorithmic properties of such classes of graphs, this identify another wide class of problems solvable in polynomial time on $H$-graphs. The most fundamental optimization problems among the problems solvable in polynomial time on $H$-graphs are Maximum Clique, Maximum Independent Set, and Minimum DominatING SET. We provide a more refined complexity analysis of these problems from the perspective of parameterized complexity. We show that Maximum IndePendent Set and Minimum Dominating Set are W[1]-hard being parameterized by the size of $H$ plus the size of the solution. On the other hand, we prove that when $H$ is a tree, then Minimum Dominating SET is fixed-parameter tractable parameterized by the size of $H$. For MAXimum CliQue we show that it admits a polynomial kernel parameterized by $H$ and the solution size.
\end{abstract}

Keywords $H$-topological intersection graphs · Mim-width · Minimal separators · Dominating set

An extended abstract of this work appeared in the proceedings of ESA 2018 [14].

Fedor V. Fomin

fedor.fomin@ii.uib.no

Extended author information available on the last page of the article 
Mathematics Subject Classification 05C62 - 05C85 - 05C69 · 68R10

\section{Introduction}

The notion of $H$-graph was introduced in the work of Bíró et al. [4] on precoloring extensions of graphs. $\mathrm{H}$-graphs nicely generalize several popular and widely studied classes of graphs. For example, the classical definition of an interval graph is as a graph which is an intersection graph ${ }^{1}$ of intervals of a line. Equivalently, a graph is interval if it is an intersection graph of some subpaths of a path. Or, equivalently, if it is an intersection graph of some subgraphs of some subdivision (which is a graph obtained by placing vertices of degree 2 on the edges) of $P_{2}$, the graph with two adjacent vertices. Similarly, every chordal graph is an intersection graph of subtrees of some tree. More generally, for a fixed graph $H$, a graph $G$ is an $H$-graph if it is an intersection graph of some connected subgraphs of some subdivision of $H$. Thus for example, an interval graph is a $P_{2}$-graph, a circular-arc graph is a $C_{2}$-graph, where $C_{2}$ is a double-edge with two endpoints, a split graph is a $K_{1, d}$-graph for some $d \geq 0$, where $K_{1, d}$ is a star with $d$ leaves, etc.

The main motivation behind the study of $H$-graphs is the following. It is wellknown that on interval, chordal, circular-arc, and other graphs with "simple" intersection models many NP-hard optimization problems are solvable in polynomial time, see e.g. the book of Golumbic [19] for an overview. It is a natural question whether at least some of these algorithmic results can be extended to more general classes of intersection graphs. Chaplick et al. [9] and Chaplick and Zeman [10] initiated the systematic study of algorithmic properties of $H$-graphs. They showed that a number of fundamental optimization problems like MAximum INDEPENDENT SET and Minimum Dominating Set are solvable in polynomial time on $H$-graphs for any fixed $H$. Most of the algorithms developed on $H$-graphs in $[9,10]$ run in time $n^{f(H)}$, where $n$ is the number of vertices in the input graph and $f$ is some function. In other words, being parameterized by $H$ most of the problems are known to be in the class XP.

Our work is driven by the following question.

- Are there generic explanations why many problems admit polynomial time algorithms on $H$-graphs?

We address the first question by proving the following combinatorial results. We show first that every $H$-graph has mim-width (a graph parameter to be defined in the corresponding section) at most $2|E(H)|+1$. Moreover, a decomposition of mimwidth $2|E(H)|+1$ can be found in polynomial time. Using known inequalities, this gives upper-bounds on the boolean-width of $H$-graphs. This combinatorial result extends the results of Belmonte and Vatshelle $[1,2]$ on the boolean-width of interval (resp. circular-arc) graphs to $H$-graphs. Together with the algorithms for a vast class of problems called LC-VSP problems [2,7] and their distance versions [22], which

\footnotetext{
${ }^{1}$ The intersection graph of a family $\mathscr{S}$ of sets has vertex set $\mathscr{S}$ and edge set $\left\{S S^{\prime}, S \cap S^{\prime} \neq \emptyset\right\}$.
} 
are solvable on $n$-vertex graphs of boolean-width $b$ in time $2^{b} \cdot n^{\mathscr{O}(1)}$, this implies immediately that all these problems are solvable in polynomial time on $H$-graphs, when $H$ is fixed. The illustrative problems solvable in polynomial time on $H$-graphs by making use of this approach are Maximum Weight IndePendent Set, Minimum Weight Dominating Set, Total Dominating Set, Induced Matching, and many others. We also obtain polynomial-time algorithms for problems related to induced paths such as Longest Induced Path and Disjoint Induced Paths using the results of Jaffke et al. [23]. Incidentally, these results demonstrate the applicability of the parameter mim-width.

Then we prove that every $n$-vertex $H$-graph has at most $(2 n+1)^{|E(H)|}+|E(H)| \cdot(2 n)^{2}$ minimal separators. ${ }^{2}$ Pipelining the bound on the number of minimal separators in $H$-graphs with meta-algorithmic results of Fomin et al. [16], we obtained another wide class of problems solvable in polynomial time on $H$-graphs. Examples of such problems are Treewidth, Minimum Feedback Vertex Set, Maximum Induced Subgraph excluding a Planar minor, and various packing problems.

All these generic algorithmic results provide XP algorithms when parameterized by the size of $H$. This brings us immediately to the second question defining the direction of our research.

- What is the parameterized complexity of the fundamental optimization problems being parameterized by the size of $H$ ?

The first steps in this direction were done by Chaplick et al. in [9] who showed that Minimum Dominating Set is fixed-parameter tractable (FPT) on $K_{1, d}$-graphs parameterized by $d$. In this paper we show that Minimum Dominating Set is W[1]-hard parameterized by the size of $H$ plus the solution size. Thus the existence of an FPT algorithm for a general graph $H$ is very unlikely. (We refer to books [11, 12] for definitions from parameterized complexity and algorithms.) We also prove a similar lower bound for Maximum IndePendent Set parameterized by the size of $H$ plus the solution size. Combined with our combinatorial results, these lower-bounds show that Maximum Independent Set and Minimum Dominating Set are also W [1]-hard when parameterized by mim-width of the input and the solution size. The technique we develop to establish lower bounds on $H$-graphs found applications beyond the topic of this paper [23, 24].

On the positive side, we show that when $H$ is a tree, then Minimum Dominating SET is FPT parameterized by the size of $H$. This significantly extends the result from [9] for stars to arbitrary trees. Furthermore, our algorithm does not require the intersection representation of the input graph to be given. We actually prove a slightly more general result, namely that Minimum Dominating SeT is FPT on chordal graphs $G$ parameterized by the leafage of the graph, i.e. the minimum number of leaves in a clique tree of $G$.

\footnotetext{
${ }^{2}$ It was reported to us by Steven Chaplick and Peter Zeman that they also obtained this result independently and that it will be included in the journal version of their paper.
} 
Table 1 Summary of algorithmic results on $H$-graphs, including the classic results on chordal graphs $(H$ is a tree)

\begin{tabular}{|c|c|c|c|c|c|}
\hline Problem & Parameters & Restrictions & Repr. & Complexity & Ref. \\
\hline Any LC-VSP-problem & $\|H\|$ & None & $\mathrm{Y}$ & $\mathrm{XP}$ & Theorem 4 \\
\hline Induced path problems & $\|H\|$ & None & $\mathrm{Y}$ & $\mathrm{XP}$ & Theorem 5 \\
\hline $\operatorname{OIS}(\mathscr{P}, t), \mathscr{P}$ is CMSOL & $\|H\|$ & None & $\mathrm{N}$ & $\mathrm{XP}$ & Corollary 3 \\
\hline \multirow[t]{5}{*}{ Dominating Set } & None & $H$ is a tree & $\mathrm{N}$ & NP-hard & {$[5]$} \\
\hline & $\|H\|$ & $H$ is a star & $\mathrm{N}$ & FPT & [9] \\
\hline & & $H$ is a tree & $\mathrm{N}$ & FPT & Theorem 9 \\
\hline & & None & $\mathrm{Y}$ & XP & [9] \\
\hline & $\|H\|+k$ & None & $\mathrm{Y}$ & W[1]-hard & Theorem 8 \\
\hline \multirow[t]{3}{*}{ INDEPENDENT SET } & None & $H$ is a tree & $\mathrm{N}$ & Polynomial & [17] \\
\hline & $\|H\|$ & None & $\mathrm{Y}$ & XP & [9] \\
\hline & $\|H\|+k$ & None & $\mathrm{Y}$ & W[1]-hard & Theorem 8 \\
\hline \multirow[t]{4}{*}{ Clique } & None & $H$ is a tree & $\mathrm{N}$ & Polynomial & [17] \\
\hline & $\|H\|$ & None & $\mathrm{Y}$ & Para-NP-hard & [10] \\
\hline & $\|H\|+k$ & None & $\mathrm{N}$ & FPT & {$[10]$} \\
\hline & & & $\mathrm{Y}$ & Poly. kernel & Theorem 10 \\
\hline
\end{tabular}

The fourth column indicates whether a representation of the input as an $H$-graph is given. For each of the mentioned problems, $k$ denotes the solution size. See Sects. 3 and 4 , for details about the first three problem

Finally we show that CLIQUE admits a polynomial kernel when parameterized by the size of $H$ plus the solution size. This strengthens the result of Chaplick and Zeman who showed that CLIQUE is FPT for such a parameterization. Our algorithmic results about $H$-graphs are summarized in Table 1.

Organization of the paper Section 2 contains the necessary definitions. In Sect. 3, we upper-bound the boolean-width of $H$-graphs and provide algorithmic applications. Section 4 is devoted to the study of minimal separators in $H$-graphs, again with algorithmic consequences. Finally, Sect. 5 contains our results on the parameterized complexity of some classic optimization problems on $H$-graphs.

\section{Definitions}

Basics All graphs in this paper are finite, undirected, loopless, and may have multiple edges. If $G$ is a graph, we respectively denote by $|G|$ and $\|G\|$ its numbers of vertices and edges (counting multiplicities). If $X$ and $Y$ are disjoint subsets of $V(G)$, $\bar{X}$ is the complement of $X$ in $V(G)$ (i.e. $\bar{X}=V(G) \backslash X), G[X]$ is the subgraph of $G$ induced by the vertices of $X$, and $G[X, Y]$ is the bipartite subgraph of $G$ with vertex set $X \cup Y$ and as edge set those edges of $G$ that have one endpoint in $X$ and the other in $Y$. Unless otherwise specified, logarithms are binary.

$H$-graphs Let $H$ be a (multi) graph. We say that a graph $G$ is an $H$-graph if there is a subdivision $H^{\prime}$ of $H$ and a collection $\mathscr{M}=\left\{M_{v}\right\}_{v \in V(G)}$ (called an H-representation or, simply, representation) of subsets of $V\left(H^{\prime}\right)$, each inducing a connected subgraph, 
such that $G$ is isomorphic to the intersection graph of $\mathscr{M}$. To avoid confusion, we refer to the vertices of $H^{\prime}$ as nodes. We also say that the nodes of $H$ are branching nodes of $H^{\prime}$ and the other nodes are subdivision nodes. If $v$ is a vertex of $G$, then $M_{v}$ is the model of $v$ in the representation $\mathscr{M}$.

For every set $A \subseteq V(G)$, we define $M_{A}=\bigcup_{v \in A} M_{v}$. For every node $u$ of $H^{\prime}$, we denote by $V_{u}$ the set of vertices of $G$ whose model contains $u$, that is,

$$
V_{u}=\left\{v \in V(G), u \in M_{v}\right\} .
$$

Parameterized Complexity We refer to the books $[11,12]$ for the detailed introduction to the field. Here we only briefly review the basic notions.

Parameterized Complexity is a two dimensional framework for studying the computational complexity of a problem. One dimension is the input size $|I|$ of an instance $I$ of the problem and the other is a parameter $k$ associated with the input. A parameterized problem is said to be fixed-parameter tractable (or FPT) if it can be solved in time $f(k) \cdot|I|^{\mathscr{O}(1)}$ for some function $f$. The parameterized complexity class FPT consists of all fixed-parameter tractable problems. A parameterized problem is in the class XP if it can be solved in time $|I|^{f(k)}$ for a function $f$. Note that if a parameterized problem is NP-hard for some fixed value of the parameter, then it is said that the problem is para-NP-hard and it cannot be in XP (and, therefore, in FPT) unless $\mathrm{P}=\mathrm{NP}$. Parameterized Complexity also provides special tools to refute the FPT algorithms under plausible complexity-theoretic assumptions. The main assumption is the conjecture that FPT $\neq \mathrm{W}[1]$ for the parameterized complexity class $\mathrm{W}[1]$ that play a central role in obtaining lower complexity bounds. The basic way to show that it is unlikely that a parameterized problem admit an FPT algorithm is to show that it is W[1]-hard using a parameterized reduction from a known W[1]-hard problem.

A kernelization for a parameterized problem is a polynomial algorithm that maps each instance $(I, k)$ of a parameterized problem with the input $I$ and parameter $k$ to an instance $\left(I^{\prime}, k^{\prime}\right)$ of the same problem such that

1. $(I, k)$ is a yes-instance if and only if $\left(I^{\prime}, k^{\prime}\right)$ is a yes-instance, and

2. $\left|I^{\prime}\right|+k^{\prime}$ is bounded by $f(k)$ for a computable function $f$.

The output $\left(I^{\prime}, k^{\prime}\right)$ is called a kernel. The function $f$ is said to be the size of the kernel. A kernel is polynomial if $f$ is polynomial. While it can be shown that every decidable parameterized problem is FPT if and only if it admits a kernel, it is unlikely that every problem in FPT has a polynomial kernel up to certain complexity assumptions. We refer to the aforementioned books and to [15] for more details.

\section{H-Graphs have Logarithmic Boolean-Width}

Boolean-width is a graph invariant that has been introduced in [7] and which is related to the number of different neighborhoods along a cut. Belmonte and Vatshelle showed in [2] that $n$-vertex interval graphs and circular-arc graphs have boolean-width $\mathscr{O}(\log n)$. In this section, we generalize their result by proving that, 
for any fixed graph $H, n$-vertex $H$-graphs have boolean-width $\mathscr{O}(\log n)$. This is done by first upper-bounding the mim-width of $H$-graphs by $\max \{1,2\|H\|\}$ (Theorem 1 ). Using the results of $[8,22,23]$, we obtain polynomial time algorithms for a vast class of optimization problems on $H$-graphs. Before we proceed with the proofs, we need to introduce some notions specific to this section.

Definition 1 A branch decomposition of a graph $G$ is a pair $(T, \delta)$ where $T$ is a full binary rooted tree (that is, every non-leaf vertex has degree 3 ) and $\delta$ is a bijection from the leaves of $T$ to the vertices of $G$. A branch decomposition $(T, \delta)$ is a caterpillar decomposition if $T$ can be obtained from a path by adding a vertex of degree one adjacent to every internal vertex. If $w \in V(T)$, let us denote by $V_{w}$ the set of vertices of $G$ in bijection with the leaves of the subtree of $T$ rooted at $w$.

Definition 2 (maximum induced matching along a cut) A set of vertices of a graph $G$ is an induced matching if it induces a disjoint union of edges. If $X \subseteq V(G)$, we denote by $\operatorname{mim}_{G}(X)$ the maximum number of edges in an induced matching of $G[X, \bar{X}]$. We drop the subscript when there is no ambiguity. If $(T, \delta)$ is a branch decomposition of $G$, we denote by $\operatorname{mim}(T, \delta)$ the maximum of $\operatorname{mim}\left(V_{w}\right)$ taken over all $w \in V(T)$ and call it the mim-width of $(T, \delta)$. The mim-width of $G$ is the minimum mim-width of a branch decomposition of $G$.

Definition 3 (neighborhood equivalence, $[2,8]$ ) Let $G$ be a graph and let $A \subseteq V(G)$. We say that two subsets $X, Y \subseteq A$ are neighborhood equivalent with respect to $A$, denoted by $X \equiv_{A} Y$, if $N(X) \cap \bar{A}=N(Y) \cap \bar{A}$.

It is not hard to see that $\equiv_{A}$ is an equivalence relation. We write nec $(A)$ for its number of equivalence classes. If $(T, \delta)$ is a branch decomposition of $G$, we denote by nec $(T, \delta)$ the maximum of nec $\left(V_{w}\right)$ and nec $\left(\overline{V_{w}}\right)$ over all $w \in V(T)$.

The following lemma relates maximum induced matchings to neighborhood equivalence.

Lemma 1 ( [2, Lemma 1]) For every n-vertex graph $G$ and $A \subseteq V(G)$, we have $\operatorname{mim}(A) \leq k$ if and only if, for every $S \subseteq A$ there is $a R \subseteq S$ such that $R \equiv_{A} S$ and $|R| \leq k$.

Definition 4 (Boolean-width) If $(T, \delta)$ is a branch decomposition of a graph $G$, the boolean-width of $(T, \delta)$, denoted by boolw $(T, \delta)$, is defined as the maximum of $\log \left(\operatorname{nec}\left(V_{w}\right)\right)$ over all $w \in V(T)$. The boolean-width of $G$, denoted by boolw $(G)$, is the minimum boolean-width of a branch decomposition of $G$.

Our results on the boolean-width of $H$-graphs follow from the next result.

Theorem 1 Let $H$ be a graph. Given any $H$-graph $G$ on $n \geq 2$ vertices, an H-representation of $G$ and the corresponding $H$-subdivision, one can compute in polynomial time a caterpillar decomposition $(T, \delta)$ with $\operatorname{mim}(T, \delta) \leq \max \{1,2\|H\|\}$. 
Proof We first assume that $H$ is connected, and explain at the end of the proof how we proceed when it is not the case. Let $F$ be the subdivision of $H$ in which $G$ can be realized and let $\left\{M_{v}\right\}_{v \in V(G)}$ be the intersection representation of $G$. We assume that both $F$ and $\left\{M_{v}\right\}_{v \in V(G)}$ are given as input of the algorithm that we describe now (in addition to $G)$.

Let us arbitrarily fix a branching node $r$ of $F$. Let $v_{1}, \ldots, v_{n}$ be an ordering of $V(G)$ by non-decreasing distance of $M_{v_{i}}$ 's to $r$.

Claim 1 For every prefix $A$ of $v_{1}, \ldots, v_{n}$ and every $S \subseteq A$, there is a set $R \subseteq S$ of size at most $\max \{1,2\|H\|\}$ such that $R \equiv_{A} S$.

Proof Let $A$ be a prefix of $v_{1}, \ldots, v_{n}$ and let $S \subseteq A$. If there is a vertex $u \in S$ such that $N(u) \cap \bar{A}=N(S) \cap \bar{A}$, we set $R=\{u\}$ and we are done. This includes the case where $G$ is a disjoint union of cliques, which happens for example when $H=K_{1}$. If such a vertex does not exist, then $H$ has more than one node; since we assume that it is connected, it also has at least one edge. Recall that $M_{A}=\bigcup_{v \in A} M_{v}$ and similarly for $M_{\bar{A}}$ and $M_{S}$. Let us consider the path $P_{e}$ corresponding to some edge $e \in E(H)$. Let $x_{1}, \ldots, x_{p}$ be the nodes of $P_{e}$ in the same order.

Let $v \in A$ and notice that since, by definition, $H\left[M_{v}\right]$ is connected, the vertex set $M_{v} \cap V\left(P_{e}\right)$ induces at most two connected components in $P_{e}$. Indeed if $M_{v} \cap V\left(P_{e}\right)$ induced more than two connected components, then one of them would not contain any endpoint of $P_{e}$, and thus this component would not be connected to other nodes of $M_{v}$ in $H\left[M_{v}\right.$ ]. Let us assume that it induces at least one connected component and let $x_{i}$ and $x_{j}$ be the first and last nodes (wrt. the ordering $x_{1}, \ldots, x_{p}$ ) of this component. If $\left\{x_{1}, \ldots, x_{i-1}\right\}$ is disjoint from $M_{\bar{A}}$, we say that $v$ is a left-protector of $P_{e}$. If $j$ is maximum among all vertices that protect the left of $P_{e}$, then $v$ is a rightmost left-protector. (Informally, it extends the most to the right.) Similarly, $v$ is a rightprotector when the right of $P_{e}$ if $\left\{x_{j+1}, \ldots, x_{p}\right\}$ is disjoint from $M_{\bar{A}}$ and is a leftmost right-protector if $i$ is minimal.

Let $Z_{e}$ be a set containing one (arbitrarily chosen) rightmost left-protector and one leftmost right-protector of $e$ if some exist, and let $R=\bigcup_{e \in E(H)} Z_{e}$. Clearly $|R| \leq 2\|H\|$. Let us now show that $N(S) \cap \bar{A} \subseteq N(R) \cap \bar{A}$. We consider a vertex $u \in N(S) \cap \bar{A}$ and we show that it also belongs to $N(R)$. Let $v$ be a neighbor of $u$ in $S$. As $u$ and $v$ are adjacent, $M_{u}$ and $M_{v}$ have non-empty intersection. Let $e$ be an edge of $H$ such that $M_{u}$ and $M_{v}$ meet on $P_{e}$, i.e. $M_{u} \cap M_{v} \cap V\left(P_{e}\right) \neq \emptyset$. Again, we denote by $x_{1}, \ldots, x_{p}$ the nodes of $P_{e}$

Claim 2 Let $w \in A$. If $M_{w}=\left\{x_{i}, \ldots, x_{j}\right\}$ for some $i, j \in\{1, \ldots p\}$ with $i \leq j$, then one of $\left\{x_{1}, \ldots x_{i-1}\right\}$ and $\left\{x_{j+1}, \ldots, x_{p}\right\}$ is disjoint from $M_{\bar{A}}$.

Proof If there are vertices $u, u^{\prime}$ of $\bar{A}$ such that $M_{u}$ and $M_{u^{\prime}}$ respectively intersect $\left\{x_{1}, \ldots x_{i-1}\right\}$ and $\left\{x_{j+1}, \ldots, x_{p}\right\}$, then one of $\operatorname{dist}_{F}\left(M_{u}, r\right)$ and $\operatorname{dist}_{F}\left(M_{u^{\prime}}, r\right)$ is smaller than $\operatorname{dist}_{F}\left(M_{w}, r\right)$. This contradicts the fact that $w \in A$ whereas $u, u^{\prime} \notin A$ and proves Claim 2. 
Claim 3 Let $w \in A$. If $M_{w}$ intersects $V\left(P_{e}\right)$ then it is a right-protector or a left-protector.

Proof By definition, if $x_{1} \in M_{w}$ then $M_{w}$ is a left-protector of $P_{e}$ (and symmetrically for the right). The case where $M_{w}$ contains none of $x_{1}$ and $x_{p}$ follows from Claim 2.

As $M_{u}$ intersects $M_{v}$ on $P_{e}$, it intersects the vertex set $C$ of one component induced by $M_{v}$ on $P_{e}$ (recall that there are either one or two such components). In the case where there are two components, we assume without loss of generality that this is the "left" one (i.e. that with smallest indices). In the case where there is one component, we assume that $v$ is a left-protector of $P_{e}$ (according to Claim $3, v$ is a leftprotector or a right-protector of $P_{e}$ ). Observe that in both cases, $v$ is a left-protector of $P_{e}$. Let $z$ be the rightmost left-protector of $P_{e}$ that belongs to $R$ and let $x_{k}, \ldots x_{k^{\prime}}$ be the nodes of the corresponding component of $P_{e}\left[M_{z} \cap V\left(P_{e}\right)\right]$ (that is, the component used in the definition of left-protector).

Notice that $C \subseteq\left\{x_{1}, \ldots, x_{k^{\prime}}\right\}$, by maximality of $z$ (informally, because it is "rightmost"). As $z$ is a left-protector, $M_{u} \cap\left\{x_{1}, \ldots, x_{k-1}\right\}=\emptyset$. Since $M_{u}$ and $C$ intersect, they intersect in $\left\{x_{k}, \ldots, x_{k^{\prime}}\right\}$. Therefore $M_{u} \cap M_{z} \neq \emptyset: z$ is adjacent to $u$. As $z \in R$, we are done.

This concludes the proof of Claim 1 .

We construct a caterpillar decomposition that follows the ordering $v_{1}, \ldots, v_{n}$ as follows. We construct a path $x_{1} \ldots x_{n}$ and $n$ vertices $y_{1}, \ldots, y_{n}$. Then we make $y_{i}$ adjacent to $x_{i}$, for every $i \in\{1, \ldots, n\}$. We define $\delta\left(y_{i}\right)=v_{i}$ for $i \in\{1, \ldots, n\}$. The root is chosen arbitrarily. According to Claim 1 and Lemma remy, this caterpillar decomposition satisfies $\operatorname{mim}(T, \delta) \leq \max \{1,2\|H\|\}$. Regarding the running time, we observe that the ordering $v_{1}, \ldots, v_{n}$ can be found by first labelling the vertices of $F$ with their distance from $r$ obtained by a BFS (in $\mathscr{O}(|F|+\|F\|)$ steps) then finding, for each $v \in V(G)$, then minimum label of a vertex in $M_{v}$ (in $O\left(\sum_{i=1}^{n}\left|M_{v}\right|\right)=O(n|F|)$ steps $)$ and finally sorting these values (in $O(n \log n)$ steps). Overall, the algorithm thus takes polynomial time in the sizes of $G$ and $F$.

We now consider the case when $H$ is not connected. Then $G$ is the disjoint union of connected graphs $G_{1}, \ldots, G_{p}$ where for every $i \in\{1, \ldots, p\}, H$ has a connected component $H^{\prime}$ such that $G_{i}$ is an $H^{\prime}$-graph. For each $G_{i}$ we can obtain an ordering $v_{1}^{i}, \ldots, v_{\left|G_{i}\right|}^{i}$ as explained above. Let $T$ be a graph obtained from the path on vertex set

$$
x_{1}^{1}, \ldots, x_{\left|G_{1}\right|}^{1}, \ldots, x_{1}^{i}, \ldots, x_{\left|G_{i}\right|}^{i}, \ldots, x_{1}^{p}, \ldots, x_{\left|G_{p}\right|}^{p}
$$

(in this order) by adding a degree one vertex $y_{j}^{i}$ adjacent to $x_{j}^{i}$, for every $i \in\{1, \ldots, p\}$ and every $j \in\left\{1, \ldots,\left|G_{i}\right|\right\}$. We root $T$ at $x_{1}^{1}$. We also define $\delta\left(y_{j}^{i}\right)=x_{j}^{i}$ for $i$ and $j$ as above. It is easy to check that $(T, \delta)$ is a caterpillar decomposition of $G$ and that

$$
\left(T\left[x_{1}^{i}, \ldots, x_{\left|G_{i}\right|}^{i}, y_{1}^{i}, \ldots, y_{\left|G_{i}\right|}^{i}\right], \delta_{\mid\left\{y_{1}^{i}, \ldots, y_{\left|G_{i}\right|}^{i}\right\}}\right)
$$


is a caterpillar decomposition of $G_{i}$ as defined at the end of the connected case. (Intuitively, we constructed a caterpillar decomposition of $G$ by attaching the caterpillar decompositions of the $G_{i}$ 's end-to-end.) As there is no edge in $G$ between vertices of distinct $G_{i}$ 's, we have $\operatorname{mim}_{G}\left(V_{x_{j}^{i}}\right)=\operatorname{mim}_{G_{i}}\left(V_{x_{j}^{i}}\right)$ and $\operatorname{mim}_{G}\left(V_{y_{j}^{i}}\right)=\operatorname{mim}_{G_{i}}\left(V_{y_{j}^{i}}\right)$, for every $i \in\{1, \ldots, p\}$ and every $j \in\left\{1, \ldots,\left|G_{i}\right|\right\}$. Hence the desired bound on the width of $(T, \delta)$ follows from the connected case.

This concludes the proof of Theorem 1.

Remark 1 We note that when $H$ is a tree, the bound in Theorem 1 can be improved to $\max \{1,\|H\|\}$. Indeed in this case, in the proof of Claim 1, for $v \in A$ and $e \in H$, the vertex set $M_{v} \cap V\left(P_{e}\right)$ induces at most one component (this follows from the choice of the sequence $\left.v_{1}, \ldots, v_{n}\right)$. Therefore $\left|Z_{e}\right| \leq 1$ for every $e \in H$ and $|R| \leq \max \{1,\|H\|\}$. The rest of the proof is identical.

The following is immediate.

Corollary 1 Let $H$ be a graph. Every $H$-graph has mim-width at most $\max \{1,2\|H\|\}$

From the definition of boolean-width, we also get the next result.

Corollary 2 Let $H$ be a graph. Every n-vertex $H$-graph with $n \geq 2$ has booleanwidth at most

$$
\max \{1,2\|H\|\} \cdot \log n \text {. }
$$

By choosing $H$ to be a single or double edge, we recover the results of [2] on the boolean-width of interval and circular-arc graphs, respectively, as special cases of Corollary 2. As proven in the same paper, there is a infinite family of interval graphs with boolean-width $\Omega(\log n)$. Apart of the degenerate case where $H$ is edgeless (in which case $H$-graphs are disjoint unions of cliques), every interval graph is an $\mathrm{H}$-graph. This shows that the bound in Corollary 2 is tight up to a constant factor.

We now provide algorithmic applications of our results. Boolean-width has been used [7] to design parameterized algorithms for the problems MAXIMUM Weight Independent Set and Minimum Weight Dominating Set. Later, invariants related to neighborhood equivalence were used in [8] as parameters of FPT algorithms for the vast class of locally checkable vertex subset and vertex partitioning problems ( LC-VSP problems), defined as follows.

Definition 5 [8] Let $\sigma$ and $\rho$ be finite or co-finite subsets of natural numbers. A subset $S$ of vertices of a graph $G$ is a $(\sigma, \rho)$-set of $\mathrm{G}$ if

$$
\forall v \in V(G),|N(v) \cap S| \in \begin{cases}\sigma & \text { if } v \in S \\ \rho & \text { otherwise. }\end{cases}
$$


A computational problem is LC-VSP if it consists in finding a minimum or maximum $(\sigma, \rho)$-set in an input graph, for some $\sigma$ and $\rho$ as above.

The class of LC-VSP problems include fundamental problems as INDEPENDENT Set, Independent Dominating Set, Total Dominating Set, and Induced MatchING. We refer to [8] for several other examples of classic computational problems expressed as LC-VSP problems. The main result of [8] is the following. While its original statement deals with the relation of $d$-neighborhood equivalence (an extension of the notion defined in Definition 3), we state it here in terms of mim-width using the direct connection between these two notions given in [2, Lemma 2].

Theorem 2 [8] For every LC-VSP problem $\Pi$, there are constants $d$ and $q$ such that $\Pi$ can be solved in time $\mathscr{O}\left(q \cdot n^{3 q d \operatorname{mim}(T, \delta)+4}\right)$ on an input graph of order $n$, if a decomposition $(T, \delta)$ of the input is given.

Furthermore, it was recently proved [22] that the distance versions of LC-VSP problems (such as $r$-INDEPENDENT SET, which asks for vertices pairwise at distance at least $r$, are also solvable in polynomial time on graphs of bounded mim-width. We refer to [22] for more details. Regarding problems that are not LC-VSP, Jaffke, Kwon, and Telle obtained polynomial-time algorithms for problems pertaining to induced paths in graphs of bounded mim-width.

Theorem 3 [23] The problems Longest Induced Path, Induced Disjoint Paths, and, for every graph $J$, the problem $J$-INDUCED SUBDIVISION ${ }^{3}$ can be solved in time $n^{\mathscr{O}(\operatorname{mim}(T, \delta))}$ on an input graph of order $n$, if a decomposition $(T, \delta)$ of the input is given.

Composing Theorem 1 with the two aforementioned results, we get the following meta-algorithmic consequences.

Theorem 4 Let $H$ be a graph and let $\Pi$ be a (distance) LC-VSP problem. Given any $\mathrm{H}$-graph, an $\mathrm{H}$-representation of it and the corresponding $\mathrm{H}$-subdivision $\mathrm{F}$, one can solve $\Pi$ in polynomial time.

By summing the running times of Theorem 1 (as detailed in its proof) and Theorem 2, we can bound the running time of the algorithm of Theorem 4 by

$$
\mathscr{O}\left(\|F\|+n|F|+q \cdot n^{6 q d\|H\|+4}\right),
$$

where $q, d>0$ are the constants depending on the problem $\Pi$ given by Theorem 2 and $n$ is the order of $G .^{4}$

\footnotetext{
3 We refer the reader to [23] for an accurate definition of these problems.

${ }^{4}$ We here assumed, for the sake of readability, that $H$ has at least one edge. In the opposite case, $G$ has a simple structure: it is a disjoint union of cliques.
} 
Theorem 5 Let $H$ and $J$ be two graphs. Given any H-graph, an H-representation of it and the corresponding H-subdivision F, one can solve any of LonGest Induced Path, Induced Disjoint Paths, and $J$-Induced Subdivision in $\mathscr{O}\left(\|F\|+n|F|+n^{\mathscr{O}(\|H\|)}\right)$ time.

\section{$4 \mathrm{H}$-Graphs have Few Minimal Separators}

Let $G$ be a graph. If $a, b \in V(G)$, we say that $X \subseteq V(G)$ is an $(a, b)$-separator if $a$ and $b$ are in distinct connected components of $G \backslash X$. It is a minimal $(a, b)$-separator if it is inclusion-wise minimal with this property. A subset of $V(G)$ is a minimal separator of $G$ if it is a minimal $(a, b)$-separator for some $a, b \in V(G)$.

The study of minimal separators is an active line of research that found many algorithmic applications (see e.g. [3, 6, 16, 25]). In general, the number of minimal separators of a graph may be as large as exponential in its number of vertices. We prove in this section that in an $H$-graph, this number is upper-bounded by a polynomial (Theorem 6). By combining this finding with meta-algorithmic results of Fomin, Todinca and Villanger [16], we deduce that a wide class of optimization problems can be solved in polynomial time on $H$-graphs (Corollary 3 ). We complement these results by providing in Lemma 2 a lower bound on the function of Theorem 6.

Theorem 6 Let $H$ be a graph. If $G$ is a H-graph, it has at most $(2|G|+1)^{\|H\|}+\|H\| \cdot(2|G|)^{2}$ minimal separators.

Proof Let $G$ be a $H$-graph. Observe that if $H$ is edgeless, then $G$ is a disjoint union of cliques and thus has either only one minimal separator, the empty set, or none if $G$ is a complete graph. Therefore we may now assume that $H$ has at least one edge.

Let $F$ be a subdivision of $H$ where $G$ can be represented as the intersection graph of $\left\{M_{v}, v \in V(G)\right\}$. For every subset $V \subseteq V(G)$, the border edges of $V$ are the edges of $F$ with one endpoint in $M_{V}$ and one endpoint in $V(F) \backslash M_{V}$. Let $R$ be the union of border edges over $\left\{M_{v}, v \in V(G)\right\}$. Observe that for every $V \subseteq V(G)$, the set of border edges of $V$ is a subset of $R$. For every edge $e \in E(F)$, we set

$$
V_{e}=\left\{v \in V(G), e \subseteq M_{v}\right\}
$$

and extend this notation to sets $S \subseteq E(F)$ as follows:

$$
V_{S}=\left\{v \in V(G), \exists s \in S, s \subseteq M_{v}\right\} .
$$

Informally, $V_{S}$ is the set of all vertices of $G$ whose models contain some edge of $S$.

Claim 4 For every minimal separator $X$ in $G$, there is a $S \subseteq R$ such that $X=V_{S}$.

Proof Let $A, B$ be two connected components of $G \backslash X$ such that $N(A)=N(B)=X$. As $X$ is an $(A, B)$-separator (i.e. $A$ and $B$ are included in the vertex sets of distinct 
connected components of $G \backslash X), M_{A} \cap M_{B}=\emptyset$. Let $S$ be the set of all border edges of $M_{A}$ that belong to some inclusion-wise minimal path that starts in $A$ and ends in $B$. As noted above, $S \subseteq R$. First we show $V_{S} \subseteq X$. Let $v \in V_{S}$. That is, $v$ is a vertex $G$ such that $M_{v} \supseteq s$ for some $s \in S$. Then $M_{v}$ contains both endpoints of $s$, one of which belongs to $M_{A}$. The vertex $v$ is adjacent to $A$ but does not belong to $A$ (as $M_{v}$ contains a vertex of $\bar{A}$ ), so it has to belong to the separator $X$. Therefore, $V_{s} \subseteq X$. Now we show $X \subseteq V_{S}$. Let $x \in X$. By definition, $x$ has a neighbor in both $A$ and $B$. Therefore, $M_{x}$ meets both $M_{A}$ and $M_{B}$. As $M_{x}$ induces a connected subgraph of $F$ and $M_{A}$ is disjoint from $M_{B}$, it contains an edge $s \in E(F)$ with one endpoint in $M_{A}$ and the other in $V(F) \backslash M_{A}$. Then $s$ is a border edge of $M_{A}$ in a minimal path from $A$ to $B$ : $x \in V_{S}$. Hence $X=V_{S}$.

From Claim 4 we can already deduce that the number of minimal separators of $G$ is at most the number of subsets of $R$. In order to obtain better bounds, we need other observations.

Claim 5 For every $V \subseteq V(G)$ such that $M_{V}$ induces a connected subgraph of $F$, and every $e \in E(H)$, the set $M_{V}$ has at most two border edges in $E\left(P_{e}\right)$. Hence, $|R| \leq 2|G| \cdot\|H\|$.

Proof Follows from the fact that $F\left[M_{V}\right]$ is connected.

Claim 6 For every minimal separator $X$ of $G$, if $S \subseteq R$ is the subset of edges of $F$ defined in the proof of Claim 4, then

- either $\left|S \cap E\left(P_{e}\right)\right| \leq 1$ for every $e \in E(H)$;

- $\operatorname{or}|S|=2$ and $S \subseteq E\left(P_{e}\right)$ for some $e \in E(H)$.

Proof Let $A$ and $B$ be as in the proof of Claim 4. According to Claim 5 and as $S$ is a subset of the border edges of $M_{A}$, we deduce $\left|S \cap E\left(P_{e}\right)\right| \leq 2$ for every $e \in E(H)$. Let us assume that $\left|S \cap E\left(P_{e}\right)\right|=2$ for some $e \in E(H)$. Let $u, u^{\prime}$ and $v, v^{\prime}$ be the endpoints of the two edges shared by $S$ and $E\left(P_{e}\right)$, respectively and in this order on the path. Then the model of one of $A$ and $B$ has its vertices in the subpath $Q$ of $P_{e}$ delimited by $u^{\prime}$ and $v$. Indeed, both $\left\{u, u^{\prime}\right\}$ and $\left\{v, v^{\prime}\right\}$ and have an endpoint that does not belong to $M_{A}$. As $M_{A}$ induces a connected subgraph of $F$, either these endpoints are $u^{\prime}$ and $v$ (intuitively, the exterior endpoints) or they are $u, v^{\prime}$ (the interior endpoints). In the first case $M_{A} \subseteq E(Q)$ and in the second one, $M_{B} \subseteq E(Q)$. From the definition of $R$, we can then conclude that $S \subseteq E\left(P_{e}\right)$ and we get $|S|=2$.

Therefore, for every minimal separator $X$ of $G$, there is a set $S \subseteq R$ such that:

1. either $\left|S \cap E\left(P_{e}\right)\right| \leq 1$ for every $e \in E(H)$;

2. or $|S|=2$ and $S \subseteq E\left(P_{e}\right)$ for some $e \in E(H)$; 
Fig. 1 A $\theta_{4}$-graph with at least $k^{4}$ minimal separators

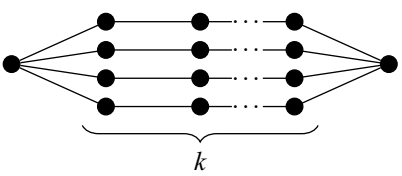

In order to upper-bound the number of possible minimal separators of $G$, it suffices to upper-bound the number of sets $S \subseteq R$ that satisfy one of the two conditions above. As noted in Claim 5, for every $e \in E(H)$ we have $R \cap E\left(P_{e}\right) \leq 2|G|$. Hence there are at most $(2|G|)^{2}$ possible choices of set $S$ that satisfy (2) for each $e \in E(H)$. We deduce that there are at most $\|H\| \cdot(2|G|)^{2}$ distinct sets $S \subseteq R$ satisfying (2). Let us now consider sets $S \subseteq R$ that satisfy (1). For every $e \in E(H)$, either $S$ contains one of the $2|G|$ edges of $R \cap E\left(P_{e}\right)$ or it does not contain any of them. This makes $2|G|+1$ possible choices for each $e \in E(H)$, and $(2|G|+1)^{\|H\|}$ in total. Consequently, $G$ has at most $(2|G|+1)^{\|H\|}+\|H\| \cdot(2|G|)^{2}$ minimal separators. This proves Theorem 6 .

For every $r \in \mathbb{N}$, let $\theta_{r}$ be the graph with 2 vertices and $r$ parallel edges. The following shows that the exponential contribution of $\|H\|$ in Theorem 6 cannot be avoided.

Lemma 2 For every $r \in \mathbb{N}$, there is a $\theta_{r}$-graph $G$ with at least $\left(\frac{|G|-2}{r}\right)^{r}$ minimal separators.

Proof Let $G$ be the graph obtained from $\theta_{r}$ by subdividing $k$ times each edge (see Fig. 1 for an example with $r=4$ ). Then $G$ is a $\theta_{r}$-graph and $|G|=k r+2$. Notice that any choice of $r$ subdivision nodes, each corresponding to a different edge of $\theta_{r}$, gives a distinct minimal separator of $G$. Hence $G$ has at least $k^{r}=\left(\frac{|G|-2}{r}\right)^{r}$ minimal separators.

Our results on minimal separators have algorithmic consequences. Let $t \in \mathbb{N}$ and let $\mathscr{P}$ be a boolean function depending on a graph and a subset of its vertices. (More formally, $\mathscr{P}(G, X)$ is a boolean value, for every graph $G$ and $X \subseteq V(G)$.) We consider the following generic problem described in [16].

Optimal Induced Subgraph For $\mathscr{P}$ AND $t$, OIS $(\mathscr{P}, t)$ for short

Input: A graph $G$

Task: $\quad$ Find sets $X \subseteq Y \subseteq V(G)$ such that $X$ is of maximum size, the induced subgraph $G[Y]$ is of treewidth at most $t$, and $\mathscr{P}(G[Y], X)$ is true.

For various choices of $\mathscr{P}$ and $t$, this generic problem corresponds to natural families of optimization meta-problems like $\mathscr{F}$-MINOR-DELETION (where $\mathscr{F}$ is a class of graphs containing at least one planar graph) whose goal is to delete a minimum number of 
vertices in order to get an $\mathscr{F}$-minor free graph $^{5}$ and INDEPENDENT $\mathscr{F}$-PACKING (where $\mathscr{F}$ is a class of connected graphs), which asks for a maximum number of disjoint copies of graphs in $\mathscr{F}$ as pairwise independent subgraphs of the input. Fomin, Todinca, and Villanger proved that when the property $\mathscr{P}$ can be expressed in Counting Monadic Second Order logic (CMSOL, see [16]), the above problem can be easily solved on classes of graphs that have a polynomial number of minimal separators.

Theorem 7 [16] For any fixed $t \in \mathbb{N}$ and CMSOL property $\mathscr{P}$, OIS $(\mathscr{P}, t)$ is solvable on an $n$-vertex graph with $s$ minimal separators in time $\mathscr{O}\left(s^{2} \cdot n^{t+4} \cdot f(t, \mathscr{P})\right)$, for some function $f$ of $t$ and $\mathscr{P}$ only.

We deduce that $\mathrm{OIS}(\mathscr{P}, t)$ can be solved in polynomial time in H-graphs:

Corollary 3 Let $H$ be a graph. For any fixed $t \in \mathbb{N}$ and CMSOL property $\mathscr{P}$, $\operatorname{OIS}(\mathscr{P}, t)$ can be solved on an $n$-vertex $H$-graph in time $n^{\mathscr{O}(\|H\|+t+4)} \cdot f(t, \mathscr{P})$, for some function fof t and $\mathscr{P}$ only.

\section{Parameterized Complexity of Basic Problems for $\mathrm{H}$-Graphs}

In this section we investigate the parameterized complexity of some basic graph problems for $H$-graphs: Dominating Set, Independent Set and Clique. First, in Sect. 5.1, we show that Dominating Set and Independent Set are W [1]-hard when parameterized by the solution size and the size of $H$. In Sect. 5.2, we show that Dominating SET is FPT when parameterized by the number of vertices of $H$ if $H$ is a tree. In fact, we show a more general result by proving that DominAting SET is FPT for chordal graphs if the problem is parameterized by the leafage of the input graph, that is, by the minimum number of leaves in a clique tree for the input graph. This result is somehow tight since Dominating Set is well-known to be W [2]-hard for split graphs when parameterized by the solution size [28]. Recall also that INDEPENDENT SET is polynomial-time solvable for chordal graphs [17, 19] and, therefore, for $H$-graphs if $H$ is a tree. Finally, in Sect. 5.3, we show that Clique admits a polynomial kernel when parameterized by the solution size and the size of $H$, in the case where the representation is given.

\subsection{Hardness of Independent Set and Dominating Set on $\boldsymbol{H}$-Graphs}

In this section we prove W[1]-hardness of Dominating Set and Independent Set for $H$-graphs (Theorem 8). Recall that Dominating Set and IndePendent Set, given a graph $G$ and a positive integer $k$, ask whether $G$ has a dominating set of size at most $k$ and independent set of size at least $k$ respectively. To show hardness, we

\footnotetext{
5 In fact, Optimal Induced Subgraph For $\mathscr{P}$ AND $t$ corresponds to the dual equivalent problem of $\mathscr{F}$ -MINOR-DELETION, which asks for a largest $\mathscr{F}$-minor free subgraph of the input.
} 


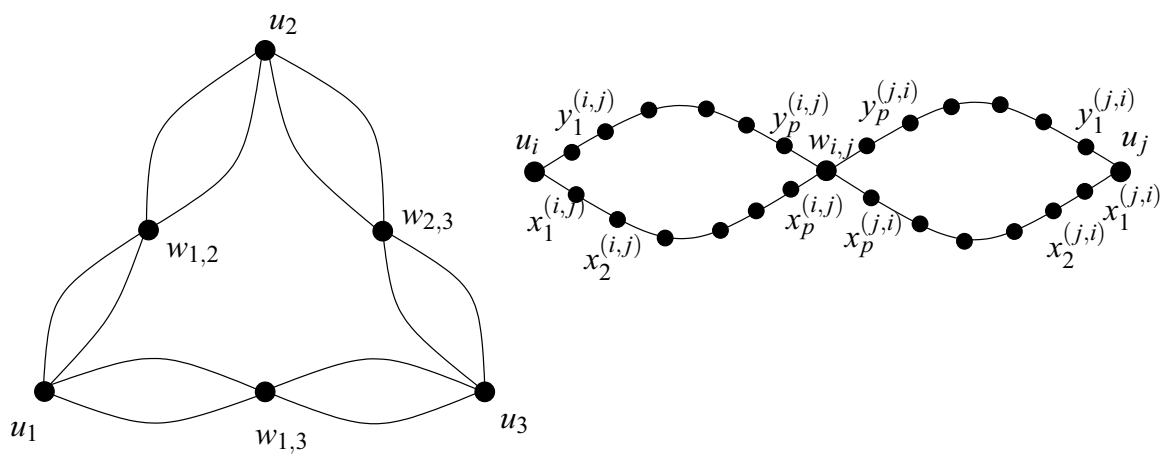

(a)

(b)

Fig. 2 The construction of $H$ for $k=3$ and the subdivision of the edges of $H$

reduce from the Multicolored Clique problem. This problem, given a graph $G$ with a $k$-partition of its vertex set $V_{1}, \ldots, V_{k}$, asks whether $G$ has a $k$-clique with exactly one vertex in each $V_{i}$ for $i \in\{1, \ldots, k\}$. The problem is well-known to be W [1]-complete when parameterized by $k[13,27]$.

Theorem 8 Dominating Set and Independent Set are W[1]-hard for H-graphs when parameterized by $k+\|H\|$ and the hardness holds even if an H-representation of $G$ is given.

Proof First, we show the W [1]-hardness for IndEPEndent SET and then explain how to modify the reduction for Dominating Set. The reduction is from Multicolored Clique.

Let $\left(G, V_{1}, \ldots, V_{k}\right)$ be an instance of Multicolored Clique. We assume that $k \geq 2$ and $\left|V_{i}\right|=p$ for $i \in\{1, \ldots, k\}$. The second assumption can be made without loss of generality because we always can add isolated vertices to the sets $V_{1}, \ldots, V_{k}$ to ensure that they have the same size. Denote by $v_{1}^{i}, \ldots, v_{p}^{i}$ the vertices of $V_{i}$ for $i \in\{1, \ldots, k\}$

We construct the multigraph $H$ as follows (see Fig. 2a)).

1. Construct $k$ nodes $u_{1}, \ldots, u_{k}$.

2. For every $i, j \in\{1, \ldots, k\}$ with $i<j$, construct a node $w_{i, j}$ and two pairs of parallel edges $u_{i} w_{i, j}$ and $u_{j} w_{i, j}$.

Note that $|H|=k(k+1) / 2$ and $\|H\|=2 k(k-1)$.

Then we construct the subdivision $H^{\prime}$ of $H$ obtained by subdividing each edge $p$ times. We denote the subdivision nodes for the 4 edges of $H$ constructed for each $i, j \in\{1, \ldots, k\}$ with $i<j$ in (ii) by $x_{1}^{(i, j)}, \ldots, x_{p}^{(i, j)}, y_{1}^{(i, j)}, \ldots, y_{p}^{(i, j)}, x_{1}^{(j, i)}, \ldots, x_{p}^{(j, i)}$ and $y_{1}^{(j, i)}, \ldots, y_{p}^{(j, i)}$ as it is shown in Fig. 2b). To simplify notations, we assume that $u_{i}=x_{0}^{(i, j)}=y_{0}^{(i, j)}, u_{j}=x_{0}^{(j, i)}=y_{0}^{(j, i)}$ and $w_{i, j}=x_{p+1}^{(i, j)}=y_{p+1}^{(i, j)}=x_{p+1}^{(j, i)}=y_{p+1}^{(j, i)}$. 


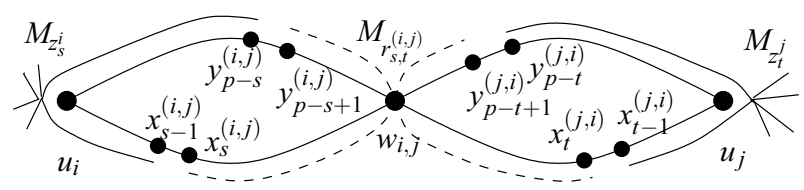

Fig. 3 The construction of $G^{\prime}$

Now we construct the $H$-graph $G^{\prime}$ by defining its $H$-representation $\mathscr{M}=\left\{M_{v}\right\}_{v \in V\left(G^{\prime}\right)}$ where the model of each vertex is a connected subset of $V\left(H^{\prime}\right)$ (see Fig. 3). Recall that $G$ is the graph of the original instance of Multicolored Clique.

1. For each $i \in\{1, \ldots, k\}$ and $s \in\{1, \ldots, p\}$, construct a vertex $z_{s}^{i}$ with the model

$$
M_{z_{s}^{i}}=\bigcup_{j \in\{1, \ldots, k\}, j \neq i}\left\{\left\{x_{0}^{(i, j)}, \ldots, x_{s-1}^{(i, j)}\right\} \cup\left\{y_{0}^{(i, j)}, \ldots, y_{p-s}^{(i, j)}\right\}\right\} .
$$

2. For each edge $v_{s}^{i} v_{t}^{j} \in E(G), s, t \in\{1, \ldots, p\}$ and $i, j \in\{1, \ldots, k\}$ with $i<j$, construct a vertex $r_{s, t}^{(l, j)}$ with the model

$$
\begin{aligned}
M_{r_{s, t}^{(i, j)}}= & \left\{x_{s}^{(i, j)}, \ldots, x_{p+1}^{(i, j)}\right\} \\
& \cup\left\{y_{p-s+1}^{(i, j)}, \ldots, y_{p+1}^{(i, j)}\right\} \\
& \cup\left\{x_{t}^{(j, i)}, \ldots, x_{p+1}^{(j, i)}\right\} \\
& \cup\left\{y_{p-t+1}^{(j, i)}, \ldots, y_{p+1}^{(j, i)}\right\} .
\end{aligned}
$$

Note that the neighborhood of $r_{s, t}^{(i, j)}$ is $\left(V_{i} \cup V_{j}\right)-v_{s}^{i}, v_{t}^{j}$. Finally, we define $k^{\prime}=k(k+1) / 2$. We claim that $\left(G, V_{1}, \ldots, V_{k}\right)$ is a yes-instance of Multicolored Clique if and only if $G^{\prime}$ has an independent set of size $k^{\prime}$. The proof is based on the following crucial property of our construction, that can be easily checked.

Claim 7 For every $i, j \in\{1, \ldots, k\}$ with $i<j$, a vertex $z_{h}^{i} \in V\left(G^{\prime}\right)$ (a vertex $\left.z_{h}^{j} \in V\left(G^{\prime}\right)\right)$ is not adjacent to a vertex $r_{s, t}^{(i, j)} \in V\left(G^{\prime}\right)$ corresponding to the edge $v_{s}^{i} v_{t}^{j} \in E(G)$ if and only if $h=s(h=t$, respectively).

We now show that $G^{\prime}$ has an independent set of size $k^{\prime}$ if $G$ has a clique of size $k$, and vice-versa. Let $\left\{v_{h_{1}}^{1}, \ldots, v_{h_{k}}^{k}\right\}$ be a clique of $G$. Consider the set

$$
I=\left\{z_{h_{1}}^{1}, \ldots, z_{h_{k}}^{k}\right\} \cup\left\{r_{h_{i}, h_{j}}^{(i, j)} \mid 0 \leq i<j \leq k\right\}
$$

of vertices of $G^{\prime}$. It is straightforward to verify using Claim 7 that $I$ is an independent set of size $k^{\prime}$ in $G^{\prime}$. 
Suppose now that $G^{\prime}$ has an independent set $I$ of size $k^{\prime}$. For each $i \in\{1, \ldots, k\}$, the set $Z_{i}=\left\{z_{h}^{i} \mid 1 \leq h \leq p\right\}$ is a clique of $G^{\prime}$, and for each $i, j \in\{1, \ldots, k\}$ with $i<j$, the set

$$
R_{i, j}=\left\{r_{s, t}^{(i, j)} \mid 1 \leq s, t \leq p, v_{s}^{i} v_{t}^{j} \in E(G)\right\}
$$

is also a clique of $G^{\prime}$. Since all these $k+\left(\begin{array}{l}k \\ 2\end{array}\right)=k(k+1) / 2=k^{\prime}$ cliques form a partition of $V\left(G^{\prime}\right)$, we have that for each $i \in\{1, \ldots, k\}$, there is a unique $z_{h_{i}}^{i} \in Z_{i} \cap I$, and for every $i, j \in\{1, \ldots, k\}$ with $i<j$, there is a unique $r_{s_{i}, s_{j}}^{(i, j)} \in R_{i, j} \cap I$. Since $r_{s_{i}, s_{j}}^{(i, j)}$ is not adjacent to $z_{h_{i}}^{i}$ and $z_{h_{j}}^{j}$, we obtain that $s_{i}=h_{i}$ and $s_{j}=h_{j}$ by Claim 7. It implies that $v_{h_{i}}^{i} v_{h_{j}}^{j} \in E(G)$. Since it holds for every $i, j \in\{1, \ldots, k\}$ with $i<j,\left\{v_{h_{1}}^{1}, \ldots, v_{h_{k}}^{k}\right\}$ is a clique in $G$.

This completes the W [1]-hardness proof for IndEPEndEnt SET. Now we explain how we modify our proof to show the W [1]-hardness of Dominating SET. This time we do not reduce from Multicolored Clique but from the Multicolored IndePendENT SET problem that, given a graph $G$ with a $k$-partition of its vertex set $V_{1}, \ldots, V_{k}$, asks whether $G$ has a independent set of size $k$ with exactly one vertex in each $V_{i}$ for $i \in\{1, \ldots, k\}$. Clearly, the W [1]-completeness of Multicolored Clique parameterized by $k[13,27]$ immediately implies the same for Multicolored IndEPENDENT SeT.

Let $\left(G, V_{1}, \ldots, V_{k}\right)$ be an instance of Multicolored Independent Set. We assume without loss of generality that $k \geq 2$ and $\left|V_{i}\right|=p$ for $i \in\{1, \ldots, k\}$. As before, denote by $v_{1}^{i}, \ldots, v_{p}^{i}$ the vertices of $V_{i}$ for $i \in\{1, \ldots, k\}$. We construct the same multigraph $H$ and its subdivision $H^{\prime}$ as above. We construct the $H$-graph $G^{\prime \prime}$ from the graph $G^{\prime}$ constructed above by adding $k$ new vertices $d_{1}, \ldots, d_{k}$ with the models $M_{d_{i}}=\left\{u_{i}\right\}$ for $i \in\{1, \ldots, k\}$.

We show that $\left(G, V_{1}, \ldots, V_{k}\right)$ is a yes-instance of Multicolored Independent Set if and only if $G^{\prime \prime}$ has a dominating set of size $k$.

Suppose that $\left\{v_{h_{1}}^{1}, \ldots, v_{h_{k}}^{k}\right\}$ is an independent set of $G$. Consider the set $D=\left\{z_{h_{1}}^{1}, \ldots, z_{h_{k}}^{k}\right\}$. By Claim 7 and the construction of $G^{\prime \prime}$, we obtain that $D$ is a dominating set of $G^{\prime \prime}$.

Let now $D$ be a dominating set of $G^{\prime \prime}$ with $|D|=k$. Note that each vertex $d_{i}$ is adjacent only to the vertices of the set $Z_{i}=\left\{z_{h}^{i} \mid 1 \leq h \leq p\right\}$ for $i \in\{1, \ldots, k\}$. It implies that for every $i \in\{1, \ldots, k\}$,

$$
D \cap\left(Z_{i} \cup\left\{d_{i}\right\}\right) \neq \emptyset .
$$

Since $Z_{i}$ is a clique, we can assume without loss of generality that $D \cap Z_{i} \neq \emptyset$ as, otherwise, we can replace $d_{i}$ in $D$ by an arbitrary vertex of $Z_{i}$. Since $Z_{i} \cap Z_{j}=\emptyset$ if $i \neq j$, we conclude that $D$ contains a unique vertex from each $Z_{i}$ and no other vertices. Let $D=\left\{z_{h_{1}}^{1}, \ldots, z_{h_{k}}^{k}\right\}$. We claim that $I=\left\{v_{h_{1}}^{1}, \ldots, v_{h_{k}}^{k}\right\}$ is an independent set of $G$. To obtain a contradiction, assume that $v_{h_{i}}^{i} v_{h_{j}}^{j} \in E(G)$ for some $i, j \in\{1, \ldots, k\}$ where $i<j$. Consider the vertex $r_{h_{i}, h_{j}}^{(i, j)}$ of $G^{\prime \prime}$. By Claim $7, r_{h_{i}, h_{j}}^{(i, j)}$ is adjacent neither to $z_{h_{i}}^{i}$ 
no $z_{h_{j}}^{j}$. Because $r_{h_{i}, h_{j}}^{(i, j)}$ is not adjacent to $z_{h_{s}}^{s}$ for any $s \in\{1, \ldots, k\}$ such that $s \neq i, j$, we have that $r_{h_{i}, h_{j}}^{(i, j)}$ is not dominated by $D$. This contradiction shows the claim and concludes the proof of Theorem 8.

Recall that we proved in Theorem 1 that for every fixed $H$, every $H$-graph has mim-width at most $2\|H\|+1$. We deduce from the negative results above the following corollary.

Corollary 4 Dominating SET and IndePendent SET are W [1]-hard when parameterized by the solution size plus the mim-width of the input.

We note that the construction in the proof of Theorem 8 has been adapted in [24] to show that the Feedback Vertex Set problem is W [1]-hard on $H$-graphs when parameterized by the solution size plus the number of edges of $H$.

\subsection{Dominating Set for T-Graphs}

In this section we show that Dominating SET is FPT for chordal graphs if the problem is parameterized by the leafage (hereafter defined) of the input graph. We stress that our algorithm does not require the intersection representation of the input graph to be given.

Let $G$ be a graph. As it is standard, we say that $u \in V(G)$ dominates $v \in V(G)$ if $v \in N_{G}[u]$ and $u$ dominates a set $W \subseteq V(G)$ if every vertex of $W$ is dominated by $u$. Respectively, a set $D \subseteq V(G)$ dominates $W \subseteq V(G)$ if every vertex of $W$ is dominated by some vertex of $D$.

Let $G$ be a graph. Let $\mathscr{K}$ be the set of (inclusion-wise) maximal cliques of $G$ and let $\mathscr{K}_{v} \subseteq \mathscr{K}$ be the set of maximal cliques containing $v \in V(G)$. A tree $T$ whose node set is $\mathscr{K}$ such that each $\mathscr{K}_{v}$ (for $v \in V(G)$ ) induces a subtree of $T$ is called a clique tree of $G$. It is well-known [18] that $G$ is a chordal graph if and only if $G$ has a clique tree $T$. Moreover, if $T$ is a clique tree of $G$, then $G$ is an intersection graph of subtrees of $T$, that is, $G$ is a $T$-graph. Conversely, if $G$ is a $T$-graph, then there is a clique tree $T^{\prime}$ of $G$ where the number of leaves of $T^{\prime}$ is at most the number of leaves of $T$. Note that a clique tree of a chordal graph is not necessarily unique. For a connected chordal graph $G$, the leafage $\ell(G)$ of $G$ is the minimum number of leaves in tree $T$ such that $G$ is a $T$-graph [26] which is also, by the above remarks, the minimum number of leaves in a clique tree of $G$. It was shown by Habib and Stacho in [20] that the leafage of a connected chordal graph $G$ can be found in polynomial time. Their algorithm also constructs a corresponding clique tree $T$ with the minimum number of leaves. In other words, given a connected chordal graph $G$, we can construct in polynomial time a clique tree $T$ with $\ell(G)$ leaves and a $T$-representation of $G$. Also, if one is given a graph $G$ that is promised to be a $T$-graph, then one can produce a clique tree $T^{\prime}$ of $G$ in polynomial time where the number of leaves of $T^{\prime}$ is at most the number of leaves of $T$.

To solve Dominating SET, we are going to use a dynamic programming algorithm over a clique tree $T^{\prime}$ of the input graph $G$. However, to do it, we have to 
Fig. 4 The construc-

tion of $X=\left\{x_{1}, x_{2}, x_{3}\right\}$

for $z_{1}, z_{2}, z_{3}, z_{4} \in D$;

$N_{T}(X)=\left\{v_{1}, \ldots, v_{5}\right\}, u_{1}=z_{1}$,

$u_{2}=z_{2}, u_{3}=z_{4}, u_{4}=u_{5}=z_{3}$,

$u_{x_{1} x_{2}}=z_{2}$ and $u_{x_{2} x_{3}}=z_{3}$

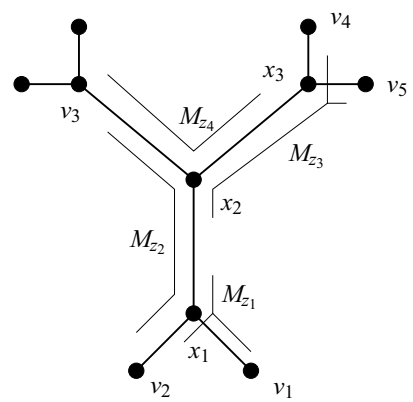

treat the vertices of $G$ whose models contain branching nodes of $T^{\prime}$ in a special way. The vertices of other type, that is, the vertices whose models contain only subdivision nodes of $T^{\prime}$ on each path corresponding to an edge of $T$, induce an interval graph and a minimum dominating set can be selected by a well-known greedy procedure (see, e.g., [19]). If the model of a vertex $v$ contains branching nodes, then this vertex can dominate various vertices whose models are in different parts of $T^{\prime}$ that can be far away from each other and, symmetrically, such a vertex can be dominated by vertices with models that are in different parts of $T^{\prime}$. To overcome these difficulties, we show that it is possible to upper bound the number of these vertices in a minimum dominating set. This allows us to guess the structure of models of the vertices in a minimum dominating sets with respect to branching vertices in them. Furthermore, we apply some reduction rules to the input graph $G$ and $T^{\prime}$ to simplify the models of the vertices of $G$. More precisely, we obtain a representation such that each model contains at most one branching node. We are paying for these reductions by switching to a special labeled variant of Dominating Set called Dominating Set Extension. Nevertheless, the obtained representation has local models and we can use it to construct a dynamic programming algorithm.

Let $T$ be a tree and let $G$ be a connected $T$-graph with its $T$-representation $\mathscr{M}=\left\{M_{v}\right\}_{v \in V(G)}$ with respect to a subdivision $T^{\prime}$ of $T$. For every non-empty $Q \subseteq V(T)$, we say that $v \in V(G)$ is a $Q$-vertex if $M_{v} \cap V(T)=Q$. If $Q=\{u\}$, we write $u$-vertex instead of $\{u\}$-vertex. Also, we denote the set of $Q$-vertices by $V_{G}(Q)$ and $V_{G}(u)$ if $Q=\{u\}$. We also denote by $V_{G}(T)$ the set of all $Q$-vertices of $G$ for every non-empty $Q \subseteq V(T)$. In other words, these are the vertices of $G$ whose models contain nodes of $T$. For every $e \in E(T), v \in V(G)$ is an $e$-vertex if $M_{v}$ contains only subdivision nodes of $T^{\prime}$ from the path in $T^{\prime}$ corresponding to $e$ in $T$. The set of $e$-vertices is denoted by $V_{G}(e)$.

We need the following lemma that allows us to upper bound the number of vertices in a minimum dominating set whose models contain given nodes of $T$.

Lemma 3 Let $\|T\| \geq 2$ and let $D$ be a minimum dominating set of $G$. Let also $X \subseteq V(T)$ be a connected set of nodes of $T$ such that 
1. for every $x \in X$, there is $u \in D$ with $x \in M_{u}$; and

2. for every $x y \in E(T)$ with $x, y \in X$, there is $u \in D$ with $x, y \in M_{u}$

(see Fig. 4). Then the set $U=\left\{u \in D \mid X \cap M_{u} \neq \emptyset\right\}$ contains at most $\left|N_{T}[X]\right|-1$ vertices.

Proof Denote by $v_{1}, \ldots, v_{s}$ the nodes of $N_{T}(X)$. To obtain a contradiction, assume that $|U| \geq\left|N_{T}[X]\right|=s+|X|$. For each $i \in\{1, \ldots, s\}$, let $u_{i} \in U$ be a vertex such that the distance between $M_{u_{i}}$ and $v_{i}$ in $T^{\prime}$ is minimum, and for each $x y \in E(T)$ with $x, y \in X$, let $u_{x y} \in U$ be an arbitrary vertex with $x, y \in M_{u_{x y}}$ (see Figure 4). Let $U^{\prime}=\left\{u_{1}, \ldots, u_{\ell}\right\} \cup\left\{u_{x y} \mid x, y \in X, x y \in E(T)\right\}$. Note that $\left|U^{\prime}\right| \leq s+\|T[X]\|=s+|X|-1=\left|N_{T}[X]\right|-1$. Since $X$ induces a subtree of $T$, we have that $\cup_{u \in U} M_{u} \subseteq \cup_{u \in U^{\prime}} M_{u}$. This immediately implies that $D^{\prime}=(D \backslash U) \cup U^{\prime}$ is a dominating set of $G$ contradicting the minimality of $D$.

In particular, since $\left|N_{T}(X)\right|$ is at most the number of leaves $\ell$, we have that $|U| \leq|X|+\ell-1$. Notice also that $\left|N_{T}[X]\right|-1=\left\|T\left[N_{T}[X]\right]\right\|$.

The next lemma gives an upper bound for the number of vertices in a minimum dominating set whose models contain nodes of $T$ (a very similar bound was given in [9, Lemma 13]).

Lemma 4 Let $D$ be a minimum dominating set of $G$ and let $\|T\| \geq 1$. Then $\left|D \cap V_{G}(T)\right| \leq 2|T|-2$.

Proof Let $D$ be a minimum dominating set of $G$. Consider the set $W$ of nodes of $T$ that are included in the models of the vertices of $D$. Let $X_{1}, \ldots, X_{r}$ be the partition of $W$ into inclusion maximal connected subsets such that for each $i \in\{1, \ldots, r\}$ and adjacent $x, y \in X_{i}$, there is $u \in D$ with $x, y \in M_{u}$. By Lemma 3 and the fact that each edge of $T$ belongs to at most two subtrees $T\left[N_{T}\left[X_{i}\right]\right]$,

$$
\left|D \cap V_{G}(T)\right| \leq \sum_{i=1}^{r}\left\|T\left[N_{T}\left(X_{i}\right)\right]\right\| \leq 2\|T\|=2|T|-2 .
$$

For an edge $e \in E(T)$, we say that $G^{\prime}$ is obtained by contracting $e$ in $T$ if $G^{\prime}$ is the (T/e)-graph with the model obtained as follows:

1. contract $x y$ in $T$ and, respectively, the $(x, y)$-path $P$ in $T^{\prime}$, and denote the node obtained from $x$ and $y$ by $z$,

2. delete all $e$-vertices of $G$,

3. for each remaining vertex $u \in V(G)$, delete from $M_{u}$ the subdivision nodes of $P$ and replace $x$ and $y$ by $z$ if at least one of these nodes is in $M_{u}$.

Note that $V\left(G^{\prime}\right) \subseteq V(G)$ and $G\left[V\left(G^{\prime}\right)\right]$ is a subgraph of $G^{\prime}$ but not necessarily induced since two vertices of $G^{\prime}$ that are not adjacent in $G$ could be adjacent in $G^{\prime}$. 
Consider a coloring $c: V_{G}(T) \rightarrow\left\{1, \ldots, 2^{|T|}\right\}$ such that for $u, v \in V_{G}(T)$, $c(u)=c(v)$ if and only if $u$ and $v$ are $Q$-vertices for the same $Q \subseteq V(T)$. The next lemmas are used to simplify the models of vertices of $G$ by contracting edges of $T$.

Lemma 5 Let $C \subseteq c\left(V_{G}(T)\right)$ and let

$$
A=\left\{x y \in E(T) \mid x, y \in M_{u} \text { for some } u \in V_{G}(T) \text { such that } c(u) \in C\right\} .
$$

Further, let $G^{\prime}$ be the graph obtained from $G$ by iteratively contracting edges of $A$ in $T$. Then for any set $D \subseteq V(G), D$ is a minimum dominating set of $G$ satisfying the condition $C=c\left(D \cap V_{G}(T)\right)$ if and only if $D$ is a minimum dominating set of $G^{\prime}$ satisfying the same condition $C=c\left(D \cap V_{G}(T)\right)$.

Proof Before we start proving the lemma, observe that $V_{G}(T) \subseteq V\left(G^{\prime}\right)$ and that $V_{G}(T)$ is the set of $Q$-vertices of $G^{\prime}$ for non-empty subsets $Q$ of the set of nodes of $T / A$. Note also that for $G^{\prime}$ and $T / A$, the coloring $c$ does not necessarily have the property that if $u$ and $v$ are $Q$-vertices of the same $Q$, then $c(u)=c(v)$.

Suppose that $D$ is a dominating set of $G$ with $C=c\left(D \cap V_{G}(T)\right)$ that has the minimum size. We claim that $D \subseteq V\left(G^{\prime}\right)$. To see it assume that there is $u \in D \backslash V\left(G^{\prime}\right)$. Then $u$ is an $e$-vertex of $G$ for some $e=x y \in A$. Then there is $v \in V_{G}(T)$ such that $x, y \in M_{v}$ and $c(v) \in C$. Since $D$ has a vertex $v^{\prime}$ with $c\left(v^{\prime}\right)=c(v)$, we have that $x, y \in M_{v^{\prime}}$. Clearly, $M_{u} \subseteq M_{v^{\prime}}$. This implies that $D \backslash\{u\}$ is a dominating set of $G$. Since $u$ is an $e$-vertex, $u$ is not colored and the obtained dominating set contains vertices with all the colors from $C$, but this contradicts the choice of $D$. Therefore, $D \subseteq V\left(G^{\prime}\right)$. Because $G\left[V\left(G^{\prime}\right)\right]$ is a subgraph of $G^{\prime}$, we have that $D$ is a dominating set of $G^{\prime}$.

Let now $D$ be a dominating set of $G^{\prime}$ with $C=c\left(D \cap V_{G}(T)\right)$ that has the minimum size. We show that $D$ is a dominating set of $G$.

First, we prove that $D$ dominates every $u \in V(G) \backslash V\left(G^{\prime}\right)$. If $u \in V(G) \backslash V\left(G^{\prime}\right)$, then $u$ is an $e$-vertex for some $e=x y \in A$. We have that there is $v \in V_{G}(T)$ such that $x, y \in M_{v}$ and $c(v) \in C$. Because $C \subseteq c\left(D \cap V_{G}(T)\right)$, there is $v^{\prime} \in D$ with $c\left(v^{\prime}\right)=c(v)$. This implies that $x, y \in M_{v^{\prime}}$ in $G$ and, therefore, $v^{\prime}$ dominates $u$.

Now we show that $D$ dominates the vertices of $V\left(G^{\prime}\right)$ in $G$. To obtain a contradiction, assume that there is $u \in V\left(G^{\prime}\right)$ that is not dominated by $D$ in $G$. As $D$ is a dominating set of $G^{\prime}$, there is $v \in D$ such that $u v \in E\left(G^{\prime}\right)$ and $u v \notin E(G)$. It follows that there are $x, y \in V(T)$ such that $x \in M_{u}, y \in M_{v}$ and for the $(x, y)$-path $P$ in $T, E(P) \subseteq A$. Let $x y^{\prime}$ be the edge of $P$ that is incident to $x$. Since $x y^{\prime} \in A$, there is $w \in V_{G}(T)$ such that $x, y \in M_{w}$ in $G$ and $c(w) \in C$. Because $C \subseteq c\left(D \cap V_{G}(T)\right)$, there is $w^{\prime} \in D$ with $c\left(w^{\prime}\right)=c(w)$. This implies that $x \in M_{w^{\prime}}$ in $G$ and, therefore, $w^{\prime}$ dominates $u$.

Lemma 6 Let $C \subseteq c\left(V_{G}(T)\right)$ and 
Fig. $5 A^{\prime}=\{x y\}$; the models of vertices of $V_{G}(T)$ with colors from $C$ are shown by solid lines and the other models are shown by dashed lines

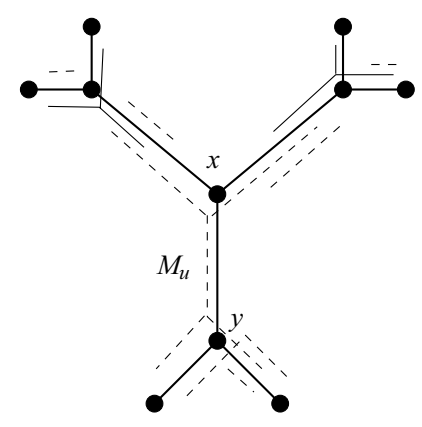

$$
\begin{gathered}
A^{\prime}=\left\{x y \in E(T) \mid x, y \in M_{u} \text { for some } u \in V_{G}(T) \text { s.t. } c(u) \notin C\right. \text { and } \\
\left.x, y \notin M_{v} \text { for all } v \in V_{G}(T) \text { s.t. } c(v) \in C\right\},
\end{gathered}
$$

and assume that for every $e \in A^{\prime}, G$ has no e-vertices (see Fig. 5 for an example). Further, let $G^{\prime}$ be the graph obtained from $G$ by iteratively contracting of edges of $A^{\prime}$ in $T$. Then for any set $D \subseteq V(G), D$ is a minimum dominating set of $G$ satisfying the condition $C=c\left(D \cap V_{G}(T)\right)$ if and only if $D$ is a dominating set of $G^{\prime}$ satisfying the same condition $C=c\left(D \cap V_{G}(T)\right)$.

Proof Observe that $V\left(G^{\prime}\right)=V(G)$, because we do not delete $e$-vertices when we contract $A^{\prime}$. Notice also that the model $M_{u}$ of a vertex $u \in V_{G}(T)$ with $c(u) \in C$ remains the same in the modified representation obtained by the contraction.

If $D$ is a dominating set of $G$ with $C=c\left(D \cap V_{G}(T)\right)$, then it is straightforward to verify that $D$ is a dominating set of $G^{\prime}$. Suppose that $D$ is a dominating set of $G^{\prime}$ with $C=c\left(D \cap V_{G}(T)\right)$. We have that $G$ is a subgraph of $G^{\prime}$. Suppose that $u, v \in V(G)$ are adjacent in $G^{\prime}$ but are not adjacent in $G$. It follows that there are $x, y \in V(T)$ such that $x \in M_{u}, y \in M_{v}$ and there is an $(x, y)$-path $P$ in $T$ such that $E(P) \subseteq A^{\prime}$. Then $c(u), c(v) \notin C$ by the definition of $A^{\prime}$. Hence, $u, v \notin D$. We obtain that for every $u, v \in V(G)$ that are adjacent in $G^{\prime}$ but are not adjacent in $G, u, v \notin D$. Hence, $D$ is a dominating set of $G$.

We say that $\mathscr{M}$ is a nice representation if $\left|M_{v} \cap V(T)\right| \leq 1$ for each $v \in V(G)$, i.e., each set $M_{v}$ contains at most one branching node of $T^{\prime}$.

We say that a $T$-representation $\mathscr{M}=\left\{M_{v}\right\}_{v \in V(G)}$ of $G$ with respect to a subdivision $T^{\prime}$ is an $r$-rooted representation if a node $r$ of $T$ is chosen to be a root. The root defines the parent-child relation on $V(T)$ and $V\left(T^{\prime}\right)$. For $x \in V(T)\left(x \in V\left(T^{\prime}\right)\right)$, we denote by $T_{x}$ ( $T_{x}^{\prime}$ respectively) the subtree of $T$ ( $T^{\prime}$ respectively) induced by $x$ and its descendants. For $x \in V(T)$ and any child $y$ of $x$, we denote by $T_{x y}$ the subtree of $T$ induced by $x, y$ and the descendants of $y$. For each $x \in V(T)$, we use $V_{x}(G)$ to denote the vertices of whose models use nodes of the subtree rooted at $x$, that is,

$$
V_{x}(G)=\left\{v \in V(G) \mid M_{v} \cap V\left(T_{x}^{\prime}\right) \neq \emptyset\right\} .
$$


For a child $y$ of $x$, we denote by $V_{x y}(G)$ the union of the set of vertices of $V_{y}(G)$ and the $x y$-vertices, that is,

$$
V_{x y}(G)=\left\{v \in V(G) \mid x \notin M_{v} \text { and } M_{v} \cap V\left(T_{x y}^{\prime}\right) \neq \emptyset\right\} .
$$

Let $\mathscr{M}=\left\{M_{v}\right\}_{v \in V(G)}$ be a nice $r$-rooted $T$-representation of $G$ with respect to $T^{\prime}$. For any vertex $x \in V\left(T^{\prime}\right)$, we denote by dist $(x)$ the distance between $r$ and $x$ in $T^{\prime}$. For every set $X \subseteq V\left(T^{\prime}\right)$, we define

$$
d_{\min }(X)=\min \{\operatorname{dist}(x) \mid x \in X\} \quad \text { and } \quad d_{\max }(X)=\max \{\operatorname{dist}(x) \mid x \in X\} .
$$

Consider an edge $e=x y \in E(T)$, where $y$ is a child of $x$, and denote by $P_{e}$ the $(x, y)$ path in $T^{\prime}$ corresponding to $e$. Consider $u, v \in V(G)$ such that $M_{u}^{e}=M_{u} \cap V\left(P_{e}\right) \neq \emptyset$ and $M_{v}^{e}=M_{v} \cap V\left(P_{e}\right) \neq \emptyset$. We write $u \preceq_{e} v$ if either

- $d_{\min }\left(M_{u}^{e}\right)<d_{\min }\left(M_{v}^{e}\right)$; or

- $d_{\min }\left(M_{u}^{e}\right)=d_{\min }\left(M_{v}^{e}\right)$ and $d_{\max }\left(M_{u}^{e}\right) \leq d_{\max }\left(M_{v}^{e}\right)$.

Respectively, $u \prec_{e} v$ if either

- $d_{\min }\left(M_{u}^{e}\right)<d_{\min }\left(M_{v}^{e}\right)$; or

- $d_{\min }\left(M_{u}^{e}\right)=d_{\min }\left(M_{v}^{e}\right)$ and $d_{\max }\left(M_{u}^{e}\right)<d_{\max }\left(M_{v}^{e}\right)$.

We consider the following auxiliary problem for $T$-graphs with nice representations. Dominating Set Extension

Input: A tree $T$ and a graph $G$ with a $T$-representation of $G$, positive integers $k$ and $d$, a labeling function $c: \bigcup_{x \in V(T)} V_{G}(x) \rightarrow \mathbb{N}$, and a collection of sets $\left\{C_{x}\right\}_{x \in V(T)}$ of size at most $d$ where for each $C_{x} \subseteq c\left(V_{G}(x)\right)$ (some sets could be empty) such that for every dominating set $D$ of $G$ of minimum size with the properties that

(a) $D$ has at most $d x$-vertices for each $x \in V(T)$,

(b) for each $x \in V(T), C_{x} \subseteq c\left(D \cap V_{G}(x)\right)$,

it holds that the number of nodes $x \in V(T)$ such that $D$ contains an $x$-vertex is maximum and for each $x \in V(T), C_{x}=c\left(D \cap V_{G}(x)\right)$.

Task: Decide whether there is a dominating set $D^{\prime}$ of $G$ of size at most $k$ containing at most $d x$-vertices for $x \in V(T)$ such that for each $x \in V(T), C_{x}=c\left(D^{\prime} \cap V_{G}(x)\right)$.

Note that Dominating Set Extension is a promise problem: we are promised that there is $D$ with the described properties but $D$ itself is not given. Moreover, the promise could be false but we are not asked to verify it.

Lemma 7 Given a nice r-rooted representation $T$ of the input graph where $T$ is a tree with at most $\ell$ leaves, Dominating SET EXTENSIon can be solved in time 
$2^{\mathscr{O}((d+\ell) \log d)} \cdot n^{\mathscr{O}(1)}$. Moreover, it can be done by an algorithm that either returns a correct yes-answer or (possibly incorrect) no-answer even if the promise is false.

Proof Let $G$ be a $T$-graph for a tree $T$ rooted in $r$ that has a nice $r$-rooted $T$-representation $\mathscr{M}$ with respect to a subdivision $T^{\prime}$ of $T$. If $G$ is disconnected, then we reduce the problem to solving Dominating Set Extension for the components of $G$. Assume from now that $G$ is connected. Let also $k$ and $d$ be positive integers, $c: \bigcup_{x \in V(T)} V_{G}(x) \rightarrow \mathbb{N}$ be a labeling function, and $\left\{C_{x}\right\}_{x \in V(T)}$ be a collection of sets where each $C_{x} \subseteq c\left(V_{G}(x)\right)$. Since $G$ is connected, we can assume without loss of generality that for every $x \in V\left(T^{\prime}\right)$, there is $v \in V(G)$ with $x \in M_{v}$. Otherwise, we can replace $T$ by its subtree or reduce the number of subdivision nodes of $T^{\prime}$ without increasing the number of leaves. We also assume that $\left|C_{x}\right| \leq d \leq\left|V_{G}(x)\right|$ as, otherwise, we have a trivial no-instance of Dominating Set Extension. We construct a dynamic programming algorithm for the problem that finds the minimum size of a dominating set $D$ of $G$ containing at most $d x$-vertices for $x \in V(T)$ such that for each $x \in V(T), C_{x}=c\left(D \cap V_{G}(x)\right)$. Our algorithm assumes that the promise is fulfilled for the considered instance of Dominating Set Extension. The algorithm uses the properties that every node of $T$ has at most $\ell$ children, i.e., the number of the children is bounded by the parameter, and for each edge $e$ of $T$, the set of $e$-vertices of $G$ composes an interval graphs for which the domination problem can be solved efficiently.

First, we construct a subroutine that solves the following auxiliary problem for each $e=x y \in E(T)$. Let $P_{e}$ be the $(x, y)$-path in $T^{\prime}$ corresponding to $e$. Let $U_{e}=\left\{v \in V(G) \mid M_{v} \cap V\left(P_{e}\right) \neq \emptyset\right\}$. For $X \subseteq U_{e}, \alpha_{e}(X)$ is the minimum size of a set $S$ of $e$-vertices of $G$ that dominates $X$; we assume that $\alpha_{e}(X)=0$ if $X=\emptyset$ and $\alpha_{e}(X)=+\infty$ if such a dominating set of $e$-vertices $S$ does not exist.

Claim 8 For every $e \in E(T)$ and $X \subseteq U_{e}, \alpha_{e}(X)$ can be computed in time $n^{\mathscr{O}(1)}$.

Proof If $X=\emptyset$, then $\alpha_{e}(X)=0$ by the definition. Assume that $X \neq \emptyset$. If there is a vertex in $X$ that is not dominated by any $e$-vertex of $G$, then we set $\alpha_{e}(X)=+\infty$. Otherwise, it is straightforward to see that $S$ exists, and we construct $S$ using the well-known greedy approach for constructing a minimum dominating set in an interval graph.

Initially, we set $S=\emptyset$ and then increase it iteratively until all the vertices of $X$ are dominated. Denote by $Y \subseteq X$ the set of vertices that are not dominated by the the current $S$. Then we do the following:

1. Find a vertex $w$ in $Y$ that is maximum with respect to the ordering $\preceq_{e}$.

2. Find a minimum with respect to $\preceq_{e} e$-vertex $v$ that dominates $w$, set $S=S \cup\{v\}$ and recompute $Y$.

3. If $Y \neq \emptyset$, then return to Step 1 .

It is straightforward to verify that the algorithm correctly computes $\alpha_{e}(X)$ in polynomial time. This proves Claim 8. 
We say that a node $x \in V(T)$ is loaded if $C_{x} \neq \emptyset$ and $x$ is unloaded otherwise. We say that a set of vertices $S$ is extendable if there is a dominating set $D$ such that

(a) $D$ has at most $d x$-vertices for $x \in V(T)$,

(b) for each $x \in V(T), C_{x} \subseteq c\left(D \cap V_{G}(x)\right)$,

(c) $D$ has the minimum size, contains $S$, and the conditions of the promise are fulfilled: the number of nodes $x \in V(T)$ such that $D$ contains an $x$-vertex is maximum and for each $x \in V(T), C_{x}=c\left(D \cap V_{G}(x)\right)$.

We are ready to explain our dynamic programming algorithm for DominAting SeT Extension. It works on $T$ starting from the leaves and moving towards the root. To avoid dealing with the root that has no parent separately, we add an artificial node $r^{\prime}$ to $T$ and $T^{\prime}$ and make $r^{\prime}$ the parent of $r$ and the new root. Observe that $r^{\prime}$ is the unique node of the tree that is not included in $M_{v}$ for any $v \in V(G)$.

We start with defining the tables of data that the algorithm stores for each $x \neq r^{\prime}$ of $T$. Let $y$ be a parent of $x$ and let $e=y x$. Consider the set $W=\left\{v_{1}, \ldots, v_{p}\right\}$ of $x$-vertices of $G$ and assume that $v_{1} \preceq_{e} \ldots \preceq_{e} v_{p}$ (note that if $x=r$ and $y=r^{\prime}$, then the ordering is arbitrary). The tables constructed in different ways for loaded and unloaded vertices, because if $x$ is loaded, then some vertices whose models contain $x$ should be included in every partial solutions and, otherwise, the partial solutions should exclude such vertices.

If $x$ is loaded, then for $x$ and $i \in\{1, \ldots, p\}$, the algorithm stores the value $\beta(x, i)$ that is either the minimum size of a set $S \subseteq V_{x}(G)$ such that

1. $v_{i} \in S$,

2. $S$ contains at most $d z$-vertices for each $z \in V\left(T_{x}\right)$,

3. for each $z \in V\left(T_{x}\right), C_{z}=c\left(S \cap V_{G}(z)\right)$, and

4. $S$ dominates all the vertices of $V_{x}(G)$,

or we may set $\beta(x, i)=+\infty$ if we detect that there is no $S \subseteq V_{x}(G)$ satisfying (1)-(4) such that $S$ is extendable. In particular, it can happen if every set $S$ satisfying (1)-(4) contradicts the promise of Dominating Set Extension.

Similarly, if $x$ is unloaded, then for $x$ and $i \in\{0, \ldots, p\}$ the algorithm stores the value $\gamma(x, i)$ that is either the minimum size of a set $S \subseteq V_{x}(G)$ such that

5. $v_{i+1}, \ldots, v_{p}$ are dominated by $S$,

6. $S$ contains at most $d z$-vertices for each $z \in V\left(T_{x}\right)$,

7. for each $z \in V\left(T_{x}\right), C_{z}=c\left(S \cap V_{G}(z)\right)$, and

8. $S$ dominates all the vertices of $V_{x}(G) \backslash\left\{v_{1}, \ldots, v_{p}\right\}$,

or we may set $\gamma(x, i)=+\infty$ if we detect that there is no $S \subseteq V_{x}(G)$ satisfying (5)-(8) such that $S$ is extendable. Similarly to $\beta(x, i)$, we do it if we detect that every set $S$ satisfying (5)-(8) contradicts the promise of Dominating Set ExtenSION. In particular, it happens when we gain by including an $x$-vertex of $G$ into a 
partial solution, that is, we can increase the number of $x$-vertices for $x \in V(T)$ in the partial solution without decreasing its size.

It also is assumed that $\beta(x, i)=+\infty$ and $\gamma(x, i)=+\infty$ if there is no $S$ that satisfies the conditions (1)-(4) or (5)-(8) respectively.

Now we explain how we compute the values $\beta(x, i)$ and $\gamma(x, i)$. First, we do it for leaves.

Computing $\beta(x, i)$ and $\gamma(x, i)$ for leaves. When $x$ is a loaded leaf of $T$, we set:

$$
\beta(x, i)= \begin{cases}\left|C_{x}\right| & \text { if } c\left(v_{i}\right) \in C_{x}, \\ +\infty & \text { otherwise } .\end{cases}
$$

When $x$ is an unloaded leaf, we set:

$$
\gamma(x, i)= \begin{cases}0 & \text { if } i=p \\ +\infty & \text { if } i<p\end{cases}
$$

Now we compute the values $\beta(x, i)$ and $\gamma(x, i)$ for non-leaves. Suppose that $x \neq r^{\prime}$ is a non-leaf node of $T$. Let $z_{1}, \ldots, z_{s}$ be the children of $x$ in $T$ and let $e_{j}=x z_{j}$ for $j \in\{1, \ldots, s\}$. Assume that the functions $\beta$ and $\gamma$ are computed for the children of $x$ depending on whether they are loaded or not.

Computing $\beta(x, i)$ for loaded $x$. Consider $z_{j}$ for $j \in\{1, \ldots, s\}$. Let $W_{j}=\left\{u_{1}, \ldots, u_{q}\right\}$ be the set of $z_{j}$-vertices of $G$. We assume that $u_{1} \preceq_{e_{j}} \ldots \preceq_{e_{j}} u_{q}$. As a first step we compute, for every $i^{\prime} \in\{1, \ldots, p\}$, the value of the auxiliary function $\delta_{j}\left(x, i^{\prime}\right)$ defined as follows, depending whether $z_{j}$ is loaded or not. Informally, $\delta_{j}\left(x, i^{\prime}\right)$ is the minimum size of a set of vertices $S_{j}$ (satisfying the constraints for considered partial solutions) of $V_{x z_{j}}(G)$ that dominate the vertices of $V_{x z_{j}}(G)$ which are not dominated by $v_{i^{\prime}}$. In particular, this means that if we add $v_{i^{\prime}}$ to a partial solution, we dominate $V_{x z_{j}}(G)$.

Case 1 The vertex $z_{j}$ is loaded. For each $i^{\prime} \in\{1, \ldots, p\}$ and $h \in\{1, \ldots, q\}$, let

$$
X_{i^{\prime}, h}=\left\{w \in V(G) \mid w \in V_{G}\left(e_{j}\right), M_{w} \cap\left(M_{v_{i^{\prime}}} \cup M_{u_{h}}\right)=\emptyset\right\} .
$$

Recall that $\alpha_{e_{j}}(X)$ is the minimum size of a set $S$ of $e_{j}$-vertices of $G$ that dominates $X$. We set

$$
\delta_{j}\left(x, i^{\prime}\right)=\min _{1 \leq h \leq q}\left\{\beta\left(z_{j}, h\right)+\alpha_{e_{j}}\left(X_{i^{\prime}, h}\right)\right\} .
$$

Case 2 The vertex $z_{j}$ is unloaded. For $i^{\prime} \in\{1, \ldots, p\}$, let

$$
Y_{i^{\prime}}=\left\{w \in V(G) \mid w \in V_{G}\left(e_{j}\right), M_{w} \cap M_{v_{i^{\prime}}}=\emptyset\right\}
$$

and for $h \in\{0, \ldots, q\}$,

$$
Y_{i^{\prime}, h}= \begin{cases}Y_{i^{\prime}} & \text { if } h=0 \\ Y_{i^{\prime}} \cup\left\{u_{h}\right\} & \text { if } h>0\end{cases}
$$


We set

$$
\delta_{j}\left(x, i^{\prime}\right)=\min _{0 \leq h \leq q}\left\{\gamma\left(z_{j}, h\right)+\alpha_{e_{j}}\left(Y_{i^{\prime}, h}\right)\right\} .
$$

We have that $\delta_{j}\left(x, i^{\prime}\right)$ is defined in both cases. Note that if $v_{i^{\prime}}$ is included in a partial solution, then it can be used to dominate vertices of $V_{x z_{j}}(G)$ for distinct $j \in\{1, \ldots, s\}$. Therefore, we extend the definition of this function on subsets of $\{1, \ldots, s\}$. For $i^{\prime} \in\{1, \ldots, p\}$ and each non-empty $J \subseteq\{1, \ldots, s\}$, we set

$$
\delta_{J}\left(x, i^{\prime}\right)=\sum_{j \in J} \delta_{j}\left(x, i^{\prime}\right) \quad \text { and } \quad \delta_{\varnothing}\left(x, i^{\prime}\right)=0 .
$$

We consider all possible partitions $\mathscr{P}=\left\{J_{1}, \ldots, J_{t}\right\}$ of $\{1, \ldots, s\}$ for all $t \in\left\{\left|C_{x}\right|, \ldots, d\right\}$, where some sets may be empty. We then consider all possible surjections $\varphi:\{1, \ldots, t\} \rightarrow C_{x}$. For every $x$ and $i \in\{1, \ldots, p\}$, we set

$$
\beta(x, i)=\min \left\{t+\sum_{h=1}^{t} \min \left\{\delta_{J_{h}}\left(x, i^{\prime}\right) \mid 1 \leq i^{\prime} \leq p, i^{\prime}=i \text { if } h=1, c\left(v_{i^{\prime}}\right)=\varphi(h)\right\} \mid \mathscr{P}, \varphi\right\}
$$

where the minimum is taken over all $\mathscr{P}$ and $\phi$; we assume to simplify notation that

$$
\min \left\{\delta_{J_{h}}\left(x, i^{\prime}\right) \mid 1 \leq i^{\prime} \leq p, i^{\prime}=i \text { if } h=1, c\left(v_{i^{\prime}}\right)=\varphi(h)\right\}=+\infty
$$

if $h \geq 2$ and there is no $i^{\prime} \in\{1, \ldots, p\}$ such that $c\left(v_{i^{\prime}}\right)=\varphi(h)$ or if $h=1$ and $\varphi(1) \neq c\left(v_{i}\right)$. The intuition behind (1) is the following. We are selecting $t x$-vertices $v_{i_{1}}, \ldots, v_{i_{t}}$ with $i=i_{1}$ (recall that $v_{i}$ should be in a solution) to include them in a partial solution. The partition $\left\{J_{1}, \ldots, J_{t}\right\}$ encodes the property that each $v_{i_{h}}$ is used to dominate some vertices of $V_{x z_{j}}(G)$ for $j \in J_{h}$. The function $\varphi$ encodes colors of the selected vertices.

Computing $\gamma(x, i)$ for unloaded $x$. Consider $z_{j}$ for each $j \in\{1, \ldots, s\}$. Let $W_{j}=\left\{u_{1}, \ldots, u_{q}\right\}$ be the set of $z_{j}$-vertices of $G$. We assume that $u_{1} \preceq_{e_{j}} \ldots \preceq_{e_{j}} u_{q}$. Recall that $W$ denotes the set of $x$-vertices. Consider also the ordering $v_{i_{1}^{j}}, \ldots, v_{i_{p}^{j}}$ of the vertices of $W$ such that $v_{i_{1}^{j}} \preceq_{e_{j}} \ldots \preceq_{e_{j}} v_{i_{p}^{j}}$. We use the following crucial property to compute $\gamma(x, i)$. Since $x$ is unloaded, the vertices of $W$ should be dominated by vertices whose models do not contain $x$ and for each $j \in\{1, \ldots, s\}$, the vertices of $V_{G}\left(x z_{j}\right)$ should be dominated by some vertices from this set. Let $S_{j} \subseteq V_{x z_{j}}(G)$ is the set of vertices that dominate the vertices of $V_{G}\left(x z_{j}\right)$ and some vertices of $W$. If $S_{j}$ is not a set of minimum size (satisfying the constraints of Dominating SET ExTENSiOn) dominating the vertices of $V_{G}\left(x z_{j}\right)$, then $S_{j}$ can be replaces by a set $S_{j}^{\prime}$ of minimum size and a vertex of $W$ that is used to dominate the vertices of $W$. This way, we obtain a partial solution that contains an $x$-vertex contradicting the promise of Dominating SET Extension. This means that to dominate the vertices of $V_{G}\left(x z_{j}\right)$, we should select a set $S_{j} \subseteq V_{x z_{j}}(G)$ of minimum size that contains a vertex whose model is at minimum possible distance from $x$. To exploit this property, we define the auxiliary functions $\eta(j)$ and $\psi(j)$ as 
follows depending on whether $z_{j}$ is loaded or not. The value of $\eta(j)$ is the minimum size of a set of vertices $S_{j}$ that dominate the vertices of $V_{G}\left(x z_{j}\right)$. The value of $\psi(j)$ is the minimum $t \in\{1, \ldots, p+1\}$ such that there is a set $S_{j}$ of size $\eta(j)$ that additionally dominates the vertices $v_{i_{t}}, \ldots, v_{i_{p}}$ (if $t=p+1$, then the vertices of $W$ are not dominated by $\left.S_{j}\right)$.

Case 1 The vertex $z_{j}$ is loaded. For each $h \in\{1, \ldots, q\}$, let

$$
X_{h}=\left\{w \in V(G) \mid w \in V_{G}\left(e_{j}\right), M_{w} \cap M_{u_{h}}=\emptyset\right\},
$$

and let

$$
\eta(j)=\min _{1 \leq h \leq q}\left(\beta\left(z_{j}, h\right)+\alpha_{e_{j}}\left(X_{h}\right)\right) .
$$

For each $t \in\{1, \ldots, p+1\}$ and $h \in\{1, \ldots, q+1\}$, denote

$$
X_{h, t}= \begin{cases}X_{h} \cup\left\{v_{i_{t}^{j}}, \ldots, v_{i_{p}^{j}}\right\} & \text { if } t \leq p \\ X_{h} & \text { if } t=p+1\end{cases}
$$

and let

$$
\psi(j)=\min \left\{t \mid 1 \leq t \leq p+1, \eta(j)=\min _{1 \leq h \leq q}\left(\beta\left(z_{j}, h\right)+\alpha_{e_{j}}\left(X_{h, t}\right)\right)\right\} .
$$

Case 2 The vertex $z_{j}$ is unloaded. For each $h \in\{0, \ldots, q\}$, let

$$
Y_{h}= \begin{cases}\left\{w \in V(G) \mid w \in V_{G}\left(e_{j}\right)\right\} & \text { if } h=0, \\ \left\{w \in V(G) \mid w \in V_{G}\left(e_{j}\right)\right\} \cup\left\{u_{1}, \ldots, u_{h}\right\} & \text { if } h \geq 1,\end{cases}
$$

and let

$$
\eta(j)=\min _{0 \leq h \leq q}\left(\gamma\left(z_{j}, h\right)+\alpha_{e_{j}}\left(Y_{h}\right)\right) .
$$

For each $t \in\{1, \ldots, p+1\}$ and $h \in\{1, \ldots, q+1\}$, denote

$$
Y_{h, t}= \begin{cases}Y_{h} \cup\left\{v_{i_{t}^{j}}, \ldots, v_{i_{p}^{j}}\right\} & \text { if } t \leq p \\ Y_{h} & \text { if } t=p+1\end{cases}
$$

and let

$$
\psi(j)=\min \left\{t \mid 1 \leq t \leq p+1, \eta(j)=\min _{0 \leq h \leq q}\left(\gamma\left(z_{j}, h\right)+\alpha_{e_{j}}\left(Y_{h, t}\right)\right)\right\} .
$$

Now for each $i \in\{0, \ldots, p\}$, we set

$$
\gamma(x, i)= \begin{cases}\sum_{j=1}^{s} \eta(j) & \text { if for } h \in\{i+1, \ldots, p\}, \text { there is } j \in\{1, \ldots, s\} \text { s.t. } v_{i_{\psi(j)}^{j}} \coprod_{e_{j}} v_{h} \\ +\infty & \text { otherwise }\end{cases}
$$

We compute $\beta$ and $\gamma$ for all nodes of $T$ except the artificial root $r^{\prime}$. The algorithm is based on the following properties of these values. 
Claim 9 If $x$ is a loaded node and $i \in\{1, \ldots, p\}$, then the following is fulfilled:

- if $\beta(x, i)<+\infty$, then there is a set $S \subseteq V_{x}(G)$ satisfying the conditions (1)-(4) of size at most $\beta(x, i)$; and

- if there is a set $S \subseteq V_{x}(G)$ of minimum size satisfying the conditions (1)-(4) that is extendable, then $|S|=\beta(x, i)$.

Similarly, if $x$ is unloaded, then for $x$ and $i \in\{0, \ldots, p\}$, the following is fulfilled:

- if $\gamma(x, i)<+\infty$, then there is a set $S \subseteq V_{x}(G)$ satisfying the conditions (5)-(8) of size at most $\gamma(x, i)$; and

- if there is a set $S \subseteq V_{x}(G)$ of minimum size satisfying the conditions (5)-(8) that is extendable, then $|S|=\mid \gamma(x, i)$.

Proof It is straightforward to verify these properties for the leaves of $T$ by the definition of $\beta$ and $\gamma$.

We use standard approach for proving correctness of dynamic programming algorithms. We assume inductively that the properties of the values of $\beta$ and $\gamma$ are fulfilled for the children of $x$ using as the base of the induction the fact that we already verified the properties of $\beta$ and $\gamma$ for the leaves of $T$.

As in the description of the algorithm, we assume that $W=\left\{v_{1}, \ldots, v_{p}\right\}$ is the set of $x$-vertices and assume that $v_{1} \preceq_{e} \ldots \preceq_{e} v_{p}$ where $e=y x$ and $y$ is the parent of $x$. In the same way, $z_{1}, \ldots, z_{s}$ are the children of $x$.

First, we prove the claim for $\beta$. Let $x$ be a loaded node and let $i \in\{1, \ldots, p\}$.

Let $\beta(x, i)<+\infty$. We show that there is a set $S \subseteq V_{x}(G)$ of size at most $\beta(x, i)$ such that

1. $v_{i} \in S$,

2. $S$ contains at most $d z$-vertices for each $z \in V\left(T_{x}\right)$,

3. for each $z \in V\left(T_{x}\right), C_{z}=c\left(S \cap V_{G}(z)\right)$, and

4. $S$ dominates all the vertices of $V_{x}(G)$.

Consider a partition $\mathscr{P}=\left\{J_{1}, \ldots, J_{t}\right\}$ of $\{1, \ldots, s\}$ and a mapping $\varphi:\{1, \ldots, t\} \rightarrow C_{x}$ for which the minimum in the right part of (1) is achieved. Further, for each $j \in\{1, \ldots, s\}$, let $i_{j}$ be a value of $i^{\prime}$ for which $\min \left\{\delta_{J_{h}}\left(x, i^{\prime}\right) \mid 1 \leq i^{\prime} \leq p, i^{\prime}=i\right.$ if $h=1, c\left(v_{i^{\prime}}\right)=\varphi(h)$ is achieved; note that $i_{1}=i$.

We define $S_{x}=\left\{v_{i_{1}}, \ldots, v_{i_{t}}\right\}$; observe that some vertices could be repeated and in this case we remove the duplicates. We have that $\left|S_{x}\right| \leq t \leq d$ and $v_{i} \in S_{x}$. Since $\varphi$ is a surjection, $C_{x}=c\left(S_{x} \cap V_{G}(x)\right)$. We obtain that (1)-(3) are fulfilled for $z=x$. Clearly, all $x$-vertices of $G$ are dominated by $S_{x}$, so (4) also holds.

Consider $z_{j}$ for $j \in\{1, \ldots, s\}$. As in the description of the algorithm, we assume that $W_{j}=\left\{u_{1}, \ldots, u_{q}\right\}$ is the set of $z_{j}$-vertices of $G$. We also assume that $u_{1} \preceq_{e_{j}} \ldots \preceq_{e_{j}} u_{q}$. Let $j \in J_{i^{\prime}}$. We consider two cases depending on whether $z_{j}$ is loaded or not. 
Case 1 The vertex $z_{j}$ is loaded. Let $h \in\{1, \ldots, q\}$ be such that $\beta\left(z_{j}, h\right)+\alpha_{e_{j}}\left(X_{i^{\prime}, h}\right)$ has the minimum value. By the inductive assumption, there is $S_{z_{j}} \subseteq V_{z_{j}}(G)$ of size at most $\beta\left(z_{j}, h\right)$ such that

1. $u_{h} \in S_{j}$,

2. $S_{z_{j}}$ contains at most $d z$-vertices for each $z \in V\left(T_{z_{j}}\right)$,

3. for each $z \in V\left(T_{z_{j}}\right), C_{z}=c\left(S_{z_{j}} \cap V_{G}(z)\right)$, and

4. $S_{z_{j}}$ dominates all the vertices of $V_{z_{j}}(G)$.

Consider $X_{i^{\prime}, h}=\left\{w \in V(G) \mid w \in V_{G}\left(e_{j}\right), M_{w} \cap\left(M_{v_{i^{\prime}}} \cup M_{u_{h}}\right)=\emptyset\right\}$. Notice that if $w$ is an $e_{j}$-vertex and $w \notin X_{i^{\prime}, h}$, then $w$ is dominated either by $v_{i^{\prime}} \in S_{x}$ or $u_{h} \in S_{z_{j}}$, i.e., $w$ is dominated by $S_{x} \cup S_{z_{j}}$. By the definition of $\alpha_{e_{j}}\left(X_{i^{\prime}, h}\right)$, there is a set $S_{x z_{j}}$ of $e_{j}$ -vertices of size $\alpha_{e_{j}}\left(X_{i^{\prime}, h}\right)$ that dominates $X_{i^{\prime}, h}$.

Case 2 The vertex $z_{j}$ is unloaded. Let $h \in\{1, \ldots, q\}$ be such that $\gamma\left(z_{j}, h\right)+\alpha_{e_{j}}\left(Y_{i^{\prime}, h}\right)$ has the minimum value.

By the inductive assumption, there is $S_{z_{j}} \subseteq V_{z_{j}}(G)$ of size at most $\gamma\left(z_{j}, h\right)$ such that

5. $u_{h+1}, \ldots, u_{q}$ are dominated by $S_{z_{j}}$,

6. $S_{z_{j}}$ contains at most $d z$-vertices for each $z \in V\left(T_{z_{j}}\right)$,

7. for each $z \in V\left(T_{z_{j}}\right), C_{z}=c\left(S \cap V_{G}(z)\right)$, and

8. $S_{z_{j}}$ dominates all the vertices of $V_{z_{j}}(G) \backslash\left\{u_{1}, \ldots, u_{q}\right\}$.

Recall that we defined

$$
\begin{aligned}
Y_{i^{\prime}} & =\left\{w \in V(G) \mid w \in V_{G}\left(e_{j}\right), M_{w} \cap M_{v_{i^{\prime}}}=\emptyset\right\}, \text { and } \\
Y_{i^{\prime}, h} & = \begin{cases}Y_{i^{\prime}} & \text { if } h=0, \\
Y_{i^{\prime}} \cup\left\{u_{h}\right\} & \text { if } h>0 .\end{cases}
\end{aligned}
$$

If $w$ is a $e_{j}$-vertex and $w \notin X_{i^{\prime}, h}$, then $w$ is dominated by $v_{i^{\prime}} \in S_{x}$, i.e., $w$ is dominated by $S_{x} \cup S_{z_{j}}$. By the definition of $\alpha_{e_{j}}\left(Y_{i^{\prime}, h}\right)$, there is a set $S_{x z_{j}}$ of $e_{j}$-vertices of size $\alpha_{e_{j}}\left(Y_{i^{\prime}, h}\right)$ that dominates $Y_{i^{\prime}, h}$. This set dominates the $e_{j}$-vertices that are not dominated by $S_{x}$ and the $z_{j}$-vertices $u_{1}, \ldots, u_{h}$ if $h \geq 1$ that are the only vertices of $V_{z_{j}}(G)$ that (possibly) are not dominated by $S_{z_{j}}$.

Now we let

$$
S=S_{x} \cup\left(\bigcup_{j=1}^{s}\left(S_{z_{j}} \cup S_{x z_{j}}\right)\right) .
$$

We have that (1)-(4) are fulfilled for $S$. It remains to notice that $|S| \leq \beta(x, i)$ by the definition.

Assume now that $S \subseteq V_{x}(G)$ is a set of minimum size satisfying (1)-(4) and $S$ is extendable. Since $S$ is a set of minimum size satisfying these conditions, as we already proved, $|S| \leq \beta(x, i)$. We prove that $|S| \geq \beta(x, i)$. 
We consider the partition $\left(S_{x}, S_{z_{1}}, \ldots, S_{z_{s}}, S_{x z_{1}}, \ldots, S_{x z_{s}}\right)$ of $S$, where $S_{z_{j}} \subseteq V_{z_{j}}(G)$ and $S_{x z_{j}}$ are $e_{j}$-vertices for each $j \in\{1, \ldots, s\}$; some sets in the partition could be empty.

Let $t=\left|S_{x}\right|$. For each $j \in\{1, \ldots, s\}$, select $v_{i(j)} \in S_{x}$ to be a maximum element of $S_{x}$ with respect to the relation $\preceq_{e_{j}}$. Observe that the selection is not necessarily unique. We consider a partition $\mathscr{P}=\left\{J_{1}, \ldots, J_{t}\right\}$ of $\{1, \ldots, s\}$ such that $j, j^{\prime} \in\{1, \ldots, s\}$ are in the same set of $\mathscr{P}$ if and only if $i(j)=i\left(j^{\prime}\right)$. We define $\varphi:\{1, \ldots, t\} \rightarrow C_{x}$ as follows. If $J_{h} \neq \emptyset$, then $\varphi(h)=c\left(v_{i(j)}\right)$ for $j \in J_{h}$. Then we extend $\varphi$ on $h \in\{1, \ldots, t\}$ with $J_{t}=\emptyset$ greedily to ensure that $\varphi$ is a surjection. Such a mapping always exists because $C_{x}=c\left(S_{x} \cap V_{G}(x)\right)$.

Consider $z_{j}$ for $j \in\{1, \ldots, s\}$. It is assumed again that $W_{j}=\left\{u_{1}, \ldots, u_{q}\right\}$ is the set of $z_{j}$-vertices of $G$. We also assume that $u_{1} \preceq_{e_{j}} \ldots \preceq_{e_{j}} u_{q}$. Let $j \in J_{i^{\prime}}$. We consider two cases depending on whether $z_{j}$ is loaded or not.

Case 1 The vertex $z_{j}$ is loaded. Let $h \in\{1, \ldots, q\}$ be such that $u_{h}$ is a minimum element of $S_{z_{j}}$ with respect to $\preceq_{e_{j}}$. By our inductive assumption, we have that $\beta\left(z_{j}, h\right) \leq\left|S_{z_{j}}\right|$, because $S_{z_{j}}$ is extendable. Consider

$$
X_{i(j), h}=\left\{w \in V(G) \mid w \in V_{G}\left(e_{j}\right), M_{w} \cap\left(M_{v_{i(j)}} \cup M_{u_{h}}\right)=\emptyset\right\} .
$$

By the definition of $i(j)$ and $h$, the vertices of $X_{i(j), h}$ are not dominated by $S_{x} \cup S_{z_{j}}$. Therefore, they are dominated by $S_{x z_{j}}$. By the definition of $\alpha_{e_{j}}$, we have that $\alpha_{e_{j}}\left(X_{i(j), h}\right) \leq\left|S_{x z_{j}}\right|$. Then

$$
\begin{aligned}
\delta_{j}(x, i(j)) & =\min _{1 \leq h^{\prime} \leq q}\left\{\beta\left(z_{j}, h^{\prime}\right)+\alpha_{e_{j}}\left(X_{i^{\prime}, h^{\prime}}\right)\right\} \\
& \leq \beta\left(z_{j}, h\right)+\alpha_{e_{j}}\left(X_{i(j), h}\right) \\
& \leq\left|S_{z_{j}}\right|+\left|S_{x z_{j}}\right| .
\end{aligned}
$$

Case 2 The vertex $z_{j}$ is unloaded. Let $h \in\{0, \ldots, q\}$ be the minimum integer such that $u_{h+1}, \ldots, u_{q}$ are dominated by $S_{z_{j}}$. Clearly, if $h>0$, then $u_{h}$ is not dominated by $S_{z_{j}}$. By the inductive assumption, we have that $\beta\left(z_{j}, h\right) \leq\left|S_{z_{j}}\right|$ as $S_{z_{j}}$ is extendable. Consider the set $Y_{i(j), h}$. By the definition of this set, we obtain that the vertices of $X_{i(j), h}$ are not dominated by $S_{x} \cup S_{z_{j}}$. Therefore, they are dominated by $S_{x z_{j}}$. By the definition of $\alpha_{e_{j}}$, we have that $\alpha_{e_{j}}\left(Y_{i(j), h}\right) \leq\left|S_{x z_{j}}\right|$. Then

$$
\begin{aligned}
\delta_{j}(x, i(j)) & =\min _{0 \leq h^{\prime} \leq q}\left\{\gamma\left(z_{j}, h^{\prime}\right)+\alpha_{e_{j}}\left(Y_{i^{\prime}, h^{\prime}}\right)\right\} \\
& \leq \gamma\left(z_{j}, h\right)+\alpha_{e_{j}}\left(Y_{i(j), h}\right) \\
& \leq\left|S_{z_{j}}\right|+\left|S_{x z_{j}}\right| .
\end{aligned}
$$

Now we combine (7) and (8) and conclude that for each $h^{\prime} \in\{1, \ldots, t\}$,

$$
\delta_{J_{h^{\prime}}}\left(x, i^{\prime}\right) \leq \sum_{j \in J_{h^{\prime}}}\left(\left|S_{z_{j}}\right|+\left|S_{x z_{j}}\right|\right),
$$


where $i^{\prime}=i(j)$ for $j \in J_{h^{\prime}}$

By (1) and (9), we obtain that

$$
\beta(x, i) \leq\left|S_{x}\right|+\sum_{j=1}^{s}\left(\left|S_{z_{j}}\right|+\left|S_{x z_{j}}\right|\right)=|S| .
$$

Our next aim is to prove Claim 9 for $\gamma$. Assume that $x$ is unloaded and let $i \in\{0, \ldots, p\}$.

We suppose that $\gamma(x, i)<+\infty$ and prove that there is a set $S \subseteq V_{x}(G)$ with $|S| \leq \gamma(x, i)$ such that

5. $v_{i+1}, \ldots, v_{p}$ are dominated by $S$,

6. $S$ contains at most $d z$-vertices for each $z \in V\left(T_{x}\right)$,

7. for each $z \in V\left(T_{x}\right), C_{z}=c\left(S \cap V_{G}(z)\right)$, and

8. $S$ dominates all the vertices of $V_{x}(G) \backslash\left\{v_{1}, \ldots, v_{p}\right\}$.

Since $\gamma(x, i)<+\infty, \gamma(x, i)=\sum_{j=1}^{s} \eta(j)$. Recall that $\eta(j)$ is computed differently depending on whether $z_{j}$ is loaded or not. We consider each $j \in\{1, \ldots, s\}$ and analyze the corresponding cases. Recall that $v_{i_{1}^{\prime}}, \ldots, v_{i_{p}^{j}}$ is the ordering of the vertices of $W$ with respect to $\preceq_{e_{j}}$.

Case 1 The vertex $z_{j}$ is loaded.

We select $t \in\{1, \ldots, p+1\}$ for which the minimum achieved in (3), that is, $\psi(j)=t$. In particular, we have that

$$
\eta(j)=\min _{1 \leq h \leq q}\left(\beta\left(z_{j}, h\right)+\alpha_{e_{j}}\left(X_{h, t}\right)\right)
$$

where

$$
X_{h, t}= \begin{cases}X_{h} \cup\left\{v_{i_{t}^{j}}, \ldots, v_{i_{p}^{j}}\right\} & \text { if } t \leq p, \\ X_{h} & \text { if } t=p+1\end{cases}
$$

and $X_{h}=\left\{w \in V(G) \mid w \in V_{G}\left(e_{j}\right), M_{w} \cap M_{u_{h}}=\emptyset\right\}$. Let $h \in\{1, \ldots, q\}$ be such that the minimum in the right part of (10) is achieved for this value.

By the inductive assumption, there is a set $S_{z_{j}} \subseteq V_{z_{j}}(G)$ of size at most $\beta\left(z_{j}, h\right)$ such that

1. $u_{h} \in S_{j}$,

2. $S_{z_{j}}$ contains at most $d z$-vertices for each $z \in V\left(T_{z_{j}}\right)$,

3. for each $z \in V\left(T_{z_{j}}\right), C_{z}=c\left(S_{z_{j}} \cap V_{G}(z)\right)$, and

4. $S_{z_{j}}$ dominates all the vertices of $V_{z_{j}}(G)$.

By the definition of $\alpha_{e_{j}}\left(X_{h, t}\right)$, there is a set of $e_{j}$-vertices $S_{x z_{j}}$ of size $\alpha_{e_{j}}\left(X_{h, t}\right)$ that dominates $X_{h, t}$. Note that $\left|S_{z_{j}}\right|+\left|S_{x z_{j}}\right| \leq \eta(j)$. Observe also that $S_{z_{j}} \cup S_{x z_{j}}$ dominates all vertices of $V_{z_{j}}(G)$ and the $e_{j}$-vertices.

Case 2 The vertex $z_{j}$ is unloaded. 
We select $t \in\{1, \ldots, p+1\}$ for which the minimum achieved in (5), that is, $\psi(j)=t$. In particular, we have that it holds

$$
\eta(j)=\min _{1 \leq h \leq q}\left(\gamma\left(z_{j}, h\right)+\alpha_{e_{j}}\left(Y_{h, t}\right)\right)
$$

where

$$
Y_{h, t}= \begin{cases}Y_{h} \cup\left\{v_{i_{t}^{\prime}}, \ldots, v_{i_{p}^{j}}\right\} & \text { if } t \leq p \\ Y_{h} & \text { if } t=p+1\end{cases}
$$

and

$$
Y_{h}= \begin{cases}\left\{w \in V(G) \mid w \in V_{G}\left(e_{j}\right)\right\} & \text { if } h=0, \\ \left\{w \in V(G) \mid w \in V_{G}\left(e_{j}\right)\right\} \cup\left\{u_{1}, \ldots, u_{h}\right\} & \text { if } h \geq 1 .\end{cases}
$$

Let $h \in\{1, \ldots, q\}$ be such that the minimum in the right part of (11) is achieved for this value. By the inductive assumption, there is a set $S_{z_{j}} \subseteq V_{z_{j}}(G)$ of size at most $\gamma\left(z_{j}, h\right)$ such that

5. $u_{h+1}, \ldots, u_{q}$ are dominated by $S_{z_{j}}$,

6. $S_{z_{j}}$ contains at most $d z$-vertices for each $z \in V\left(T_{z_{j}}\right)$,

7. for each $z \in V\left(T_{z_{j}}\right), C_{z}=c\left(S_{z_{j}} \cap V_{G}(z)\right)$, and

8. $S_{z_{j}}$ dominates all the vertices of $V_{x}(G) \backslash\left\{u_{1}, \ldots, u_{q}\right\}$.

By the definition of $\alpha_{e_{j}}\left(Y_{h, t}\right)$, there is a set of $e_{j}$ vertices $S_{x z_{j}}$ of size $\alpha_{e_{j}}\left(Y_{h, t}\right)$ that dominates $Y_{h, t}$. We have that $\left|S_{z_{j}}\right|+\left|S_{x z_{j}}\right| \leq \eta(j)$. Notice that $S_{z_{j}} \cup S_{x z_{j}}$ dominates all vertices of $V_{z_{j}}(G)$ and the $e_{j}$-vertices.

Now we define

$$
S=\cup_{j=1}^{s}\left(S_{z_{j}} \cup S_{x z_{j}}\right)
$$

We have that

$$
|S|=\sum_{j=1}^{s}\left(\left|S_{z_{j}}\right|+\left|S_{x z_{j}}\right|\right) \leq \sum_{j=1}^{s} \eta(j)=\gamma(x, i)
$$

By the definition of $S$, we have that (6)-(8) are fulfilled. To show (5), recall that $\gamma(x, i)<+\infty$. Then for each $h \in\{i+1, \ldots, p\}$, there is $j \in\{1, \ldots, s\}$ such that $v_{i_{\psi(j)}^{j}} \preceq_{e_{j}} v_{h}$ and, therefore, $v_{h}$ is dominated by $S_{x z_{j}}$.

Assume now that $S \subseteq V_{x}(G)$ is a set of minimum size satisfying (5)-(8) for $x$ and $i \in\{1, \ldots, p\}$ and $S$ is extendable. Because $S$ is a set of minimum size satisfying these conditions, $|S| \leq \gamma(x, i)$. We show that $|S| \geq \gamma(x, i)$.

We consider the partition $\left(S_{z_{1}}, \ldots, S_{z_{s}}, S_{x z_{1}}, \ldots, S_{x z_{s}}\right)$ of $S$, where $S_{z_{j}} \subseteq V_{z_{j}}(G)$ and $S_{x z_{j}}$ are $e_{j}$-vertices for $j \in\{1, \ldots, s\}$; some sets in the partition could be empty. Note that since $S$ is extendable, all the sets in the partition are extendable as well. 
First, we show that $\eta(j) \leq\left|S_{z_{j}}\right|+\left|S_{x z_{j}}\right|$ for $j \in\{1, \ldots, s\}$.

If $x_{j}$ is loaded then $\eta(j)$ is computed by (2). Let $h \in\{1, \ldots, q\}$ be such that $u_{h}$ is a minimum with respect to $\preceq_{e_{j}}$ vertex in $S_{z_{j}}$. By induction, $\left|S_{z_{j}}\right| \geq \beta\left(z_{j}, h\right)$. Since the vertices of $G$ that are in $X_{h}=\left\{w \in V(G) \mid w \in V_{G}\left(e_{j}\right), M_{w} \cap M_{u_{h}}=\emptyset\right\}$ are not dominated by $S_{z_{j}}$, they are dominated by $S_{x z_{j}}$. By the definition of $\alpha_{e_{j}}\left(X_{h}\right),\left|S_{x z_{j}}\right| \geq \alpha_{e_{j}}\left(X_{h}\right)$. Therefore, $\eta(j) \leq \beta\left(z_{j}, h\right)+\alpha_{e_{j}}\left(X_{h}\right) \leq\left|S_{z_{j}}\right|+\left|S_{x z_{j}}\right|$.

If $z_{j}$ is unloaded, then $\eta(j)$ is computed by (4). We find minimum $h \in\{0, \ldots, q\}$ such that $u_{h+1}, \ldots, u_{q}$ are dominated by $S_{z_{j}}$. By induction, $\left|S_{z_{j}}\right| \geq \gamma\left(z_{j}, h\right)$ We consider

$$
Y_{h}= \begin{cases}\left\{w \in V(G) \mid w \in V_{G}\left(e_{j}\right)\right\} & \text { if } h=0, \\ \left\{w \in V(G) \mid w \in V_{G}\left(e_{j}\right)\right\} \cup\left\{u_{1}, \ldots, u_{h}\right\} & \text { if } h \geq 1,\end{cases}
$$

and observe that the vertices of $Y_{h}$ are not dominated by $S_{z_{j}}$. Hence, the vertices of $Y_{h}$ are dominated by $S_{x z_{j}}$. By the definition of $\alpha_{z_{j}}\left(Y_{h}\right),\left|S_{x z_{j}}\right| \geq \alpha\left(Y_{h}\right)$. Hence, $\eta(j) \leq \gamma\left(z_{j}, h\right)+\alpha_{e_{j}}\left(X_{h}\right) \leq\left|S_{z_{j}}\right|+\left|S_{x z_{j}}\right|$.

Since $\eta(j) \leq\left|S_{z_{j}}\right|+\left|S_{x z_{j}}\right|$ for all $j \in\{1, \ldots, s\}$, we have that if $\gamma(x, i)=\sum_{j=1}^{s} \eta(j)$, then

$$
\gamma(x, i)=\sum_{j=1}^{s} \eta(j) \leq \sum_{j=1}^{s}\left(\left|S_{z_{j}}\right|+\left|S_{x z_{j}}\right|\right)=|S| .
$$

Suppose now that $\gamma(x, i) \neq \sum_{j=1}^{s} \eta(j)$. By (6), we have that there is $h^{\prime} \in\{i+1, \ldots, p\}$ such that $v_{h^{\prime}} \prec_{e_{j}} v_{i_{\psi(j)}^{j}}$ for all $j \in\{1, \ldots, s\}$. The vertex $v_{h^{\prime}}$ is dominated by $S$ by the condition (v). Assume that $v_{h^{\prime}}$ is dominated by $S_{z_{j}} \cup S_{x z_{j}}$ for $j \in\{1, \ldots, s\}$. Let $v_{h^{\prime}}=v_{i_{t}^{t}}$ according to the ordering of $x$-vertices with respect to $\preceq_{e_{j}}$. We again consider two cases.

Case 1 The vertex $z_{j}$ is loaded.

By (3), $\quad \eta(j)<\min _{1 \leq h \leq q}\left(\beta\left(z_{j}, h\right)+\alpha_{e_{j}}\left(X_{h, t}\right)\right), \quad$ where $X_{h}=\left\{w \in V(G) \mid w \in V_{G}\left(e_{j}\right), M_{w} \cap M_{u_{h}}=\emptyset\right\}$ and

$$
X_{h, t}= \begin{cases}X_{h} \cup\left\{v_{i_{t}^{j}}, \ldots, v_{i_{p}^{j}}\right\} & \text { if } t \leq p, \\ X_{h} & \text { if } t=p+1 .\end{cases}
$$

Let $h \in\{1, \ldots, q\}$ be such that $u_{h}$ is a minimum with respect to $\preceq_{e_{j}}$ vertex in $S_{z_{j}}$. By induction, $\left|S_{z_{j}}\right| \geq \beta\left(z_{j}, h\right)$. Since the $e_{j}$-vertices of $G$ that are in $X_{h, t}$ are not dominated by $S_{z_{j}}$, they are dominated by $S_{x z_{j}}$. By the definition of $\alpha_{e_{j}}\left(X_{h, t}\right),\left|S_{x z_{j}}\right| \geq \alpha_{e_{j}}\left(X_{h, t}\right)$. This means that $\eta(j)<\left|S_{z_{j}}\right|+\left|S_{x z_{j}}\right|$.

Let $h^{*} \in\{1, \ldots, q\}$ be such that the minimum in the right part of (10) is achieved for this value, that is, $\eta(j)=\beta\left(z_{j}, h\right)+\alpha_{e_{j}}\left(X_{h^{*}}\right)$. By the inductive assumption, there is a set $S_{z_{j}}^{\prime} \subseteq V_{z_{j}}(G)$ of size at most $\beta\left(z_{j}, h^{*}\right)$ such that

1. $u_{h^{*}} \in S_{j}^{\prime}$

2. $S_{z_{j}}^{\prime}$ contains at most $d z$-vertices for each $z \in V\left(T_{z_{j}}\right)$, 
3. for each $z \in V\left(T_{z_{j}}\right), C_{z}=c\left(S_{z_{j}} \cap V_{G}(z)\right)$, and

4. $S_{z_{j}}^{\prime}$ dominates all the vertices of $V_{z_{j}}(G)$.

By the definition of $\alpha_{e_{j}}\left(X_{h^{*}}\right)$, there is a set of $e_{j}$ vertices $S_{x z_{j}}^{\prime}$ of size $\alpha_{e_{j}}\left(X_{h^{*}}\right)$ that dominates $X_{h^{*}}$. Note that $\left|S_{z_{j}}\right|+\left|S_{x z_{j}}\right| \leq \eta(j)$. Observe also that $S_{z_{j}}^{\prime} \cup S_{x z_{j}}^{\prime}$ dominates all vertices of $V_{z_{j}}(G)$ and the $e_{j}$-vertices. Let $S^{\prime}=S_{z_{j}}^{\prime} \cup S_{x z_{j}}^{\prime} \cup\left\{v_{1}\right\}$. This set dominates all the vertices of $V_{x}(G)$. Note that $\left|S^{\prime}\right| \leq\left|S_{z_{j}} \cup S_{x z_{j}}\right|$.

Case 2 The vertex $z_{j}$ is unloaded.

By (5), $\eta(j)<\min _{1 \leq h \leq q}\left(\gamma\left(z_{j}, h\right)+\alpha_{e_{j}}\left(Y_{h, t}\right)\right)$, where

$$
Y_{h, t}= \begin{cases}Y_{h} \cup\left\{v_{i_{t}^{j}}, \ldots, v_{i_{p}^{j}}\right\} & \text { if } t \leq p \\ Y_{h} & \text { if } t=p+1,\end{cases}
$$

and

$$
Y_{h}= \begin{cases}\left\{w \in V(G) \mid w \in V_{G}\left(e_{j}\right)\right\} & \text { if } h=0 \\ \left\{w \in V(G) \mid w \in V_{G}\left(e_{j}\right)\right\} \cup\left\{u_{1}, \ldots, u_{h}\right\} & \text { if } h \geq 1\end{cases}
$$

Let $h \in\{0, \ldots, q\}$ be the minimum index such that $u_{h+1}, \ldots, u_{q}$ are dominated by $S_{z_{j}}$. By induction, $\left|S_{z_{j}}\right| \geq \gamma\left(z_{j}, h\right)$. Since the vertices of $G$ that are in $Y_{h, t}$ are not dominated by $S_{z_{j}}$, they are dominated by $S_{x z_{j}}$. By the definition of $\alpha_{e_{j}}\left(X_{h, t}\right),\left|S_{x z_{j}}\right| \geq \alpha_{e_{j}}\left(X_{h, t}\right)$. It means that $\eta(j)<\left|S_{z_{j}}\right|+\left|S_{x z_{j}}\right|$.

Let $h^{*} \in\{1, \ldots, q\}$ be such that the minimum in the right part of (11) is achieved for this value, that is, $\eta(j)=\gamma\left(z_{j}, h^{*}\right)+\alpha_{e_{j}}\left(Y_{h^{*}}\right)$. By the inductive assumption, there is a set $S_{z_{j}}^{\prime} \subseteq V_{z_{j}}(G)$ of size at most $\gamma\left(z_{j}, h^{*}\right)$ such that

5. $u_{h^{*}+1}, \ldots, u_{q}$ are dominated by $S_{z_{j}}$,

6. $S_{z_{j}}$ contains at most $d z$-vertices for each $z \in V\left(T_{z_{j}}\right)$,

7. for each $z \in V\left(T_{z_{j}}\right), C_{z}=c\left(S_{z_{j}} \cap V_{G}(z)\right)$, and

8. $S_{z_{j}}$ dominates all the vertices of $V_{x}(G) \backslash\left\{u_{1}, \ldots, u_{q}\right\}$.

By the definition of $\alpha_{e_{j}}\left(X_{h}\right)$, there is a set of $e_{j}$ vertices $S_{x z_{j}}^{\prime}$ of size $\alpha_{e_{j}}\left(Y_{h^{*}}\right)$ that dominates $Y_{h^{*}}$. Note that $\left|S_{z_{j}}\right|+\left|S_{x z_{j}}\right| \leq \eta(j)$. Observe also that $S_{z_{j}}^{\prime} \cup S_{x z_{j}}^{\prime}$ dominates all vertices of $V_{z_{j}}(G)$ and the $e_{j}$-vertices. Let $S^{\prime}=S_{z_{j}}^{\prime} \cup S_{x z_{j}}^{\prime} \cup\left\{v_{1}\right\}$. This set dominates all the vertices of $V_{x}(G)$. Note that $\left|S^{\prime}\right| \leq\left|S_{z_{j}} \cup S_{x z_{j}}\right|$.

Now we use the set $S^{\prime}$ obtained in both cases to obtain a contradiction to the extendability of $S$. The set $S$ is extendable, that is, there is a dominating set $D$ of $G$ such that

(a) $D$ has at most $d x$-vertices for $x \in V(T)$,

(b) for each $x \in V(T), C_{x} \subseteq c\left(D \cap V_{G}(x)\right)$, 
that has the minimum size and contains $C$ and the conditions of the promise is fulfilled: the number of nodes $z \in V(T)$ such that $D$ contains an $z$-vertex is maximum and for each $z \in V(T), C_{z}=c\left(D \cap V_{G}(z)\right)$. Let $D^{\prime}=\left(D \backslash\left(S_{z_{i}} \cup S_{x z_{j}}\right)\right) \cup S^{\prime}$. It is straightforward to see that $D$ is a dominating set and $\left|D^{\prime}\right| \leq|D|$. We also have that (a) and (b) are fulfilled for $D^{\prime}$, because of (ii'), (iii'), (vi') and (vii'), but this contradicts the condition that the number of nodes $x \in V(T)$ such that $D$ contains an $x$-vertex is maximum because $D^{\prime}$ contains the $x$-vertex $v_{1}$. Hence, $\gamma(x, i) \neq \sum_{j=1}^{s} \eta(j)$ and this completes the proof of the claim that $|S| \geq \gamma(x, i)$. We thus proved Claim 9.

Now we are ready to complete the description of our algorithm for DominATING SET EXTENSION. The algorithm computes the table of values of $\beta(r, i)$ if $r$ is loaded and the table of values of $\gamma(r, i)$ if $r$ is unloaded. If $r$ is loaded, we find the minimum value $\beta\left(r, i^{*}\right)$ in the table for $i$. By Claim 9 , if $\beta\left(r, i^{*}\right)<+\infty$, then $G$ has a dominating set $S$ of size at most $\beta\left(r, i^{*}\right)$ containing at most $d x$-vertices for $x \in V(T)$ such that for each $x \in V(T), C_{x}=c\left(S \cap V_{G}(x)\right)$. Moreover, if the promise is true, then $\beta\left(r, i^{*}\right)<+\infty$ and the minimum size of $S$ is $\beta\left(r, i^{*}\right)$. If $r$ is unloaded, then we consider $\gamma(r, 0)$ in the table for $r$. By Claim 9, if $\gamma(r, 0)<+\infty$, then $G$ has a dominating set $S$ of size at most $\gamma(r, 0)$ containing at most $d x$-vertices for $x \in V(T)$ such that for each $x \in V(T)$, $C_{x}=c\left(S \cap V_{G}(x)\right)$, and if the promise is true, then $\gamma(r, 0)<+\infty$ and the minimum size of $S$ is $\gamma(r, 0)$. It remains to check whether $\beta\left(r, i^{*}\right) \leq k$ or $\gamma(r, 0) \leq k$ respectively and return the answer.

To evaluate the running time, observe that to compute $\beta(x, i)$, we consider all possible partitions $\mathscr{P}=\left\{J_{1}, \ldots, J_{t}\right\}$ for $1 \leq t \leq d$ of $\{0,1, \ldots, s\}$ into non-empty sets such that $0 \in J_{1}$ where $s$ is the number of children of $x$. Since $s \leq \ell$, we have that the number of partitions is $2^{\mathscr{O}(\ell \log d)}$. Then for each partition $\mathscr{P}=\left\{J_{1}, \ldots, J_{t}\right\}$, we consider all possible surjections $\varphi:\{1, \ldots, t\} \rightarrow C_{x}$. Since $t \leq d$ and each $\left|C_{x}\right| \leq d$, there are $2^{\mathscr{O}(d \log d)}$ choices of $\varphi$. Because each value of $\alpha$ and $\gamma$ can be computed in polynomial time, it implies that the total running time of the algorithms is $2^{\mathscr{O}((\ell+d) \log d)} n^{\mathscr{O}(1)}$. This finishes the proof of Lemma 7 about DominAting SeT EXTENSION.

Now we are ready to prove the main theorem of the section.

Theorem 9 Dominating SET can be solved in time $2^{\mathscr{O}\left(\ell^{2}\right)} \cdot n^{\mathscr{O}(1)}$ for connected chordal graphs with leafage at most $\ell$.

Proof Let $(G, k)$ be an instance of Dominating SET where $G$ is a connected chordal graph.

We use the algorithm of Habib and Stacho [20] to compute its leafage $\ell(G)$. If $\ell(G)>\ell$, we stop and return a no-answer. Otherwise, we consider the clique tree $T^{\prime}$ of $G$ constructed by the algorithm. If $|T|=1$, then $G$ is a complete graph and Dominating Set has a straightforward solution. Let $\|T\| \geq 1$, that is, $\ell(G) \geq 2$. We construct the tree $T$ from $T^{\prime}$ by dissolving nodes of degree two, that is, for a node $x$ of degree two with the neighbors $y$ and $z$, we delete $x$ and make $y$ and $z$ adjacent. Observe that since $T$ is a tree with at most $\ell$ leaves that has no node of degree two, 
$|T| \leq 2 \ell-2$. We have that $G$ is a $T$-graph. Note also that the algorithm of Habib and Stacho [20] gives us a $T$-representation $\mathscr{M}=\left\{M_{v}\right\}_{v \in V(G)}$ where $M_{v} \in V\left(T^{\prime}\right)$ for $v \in V(G)$.

We consider the $2^{|T|}-1 \leq 2^{2 \ell-2}-1$ non-empty subsets of $V(T)$ and construct a coloring $c: V_{G}(T) \rightarrow\left\{1, \ldots, 2^{|T|}\right\}$ such that for $u, v \in V_{G}(T), c(u)=c(v)$ if and only if $u$ and $v$ are $Q$-vertices for the same $Q \subseteq V(T)$.

By Lemma 4, a minimum dominating set of $G$ contains at most $2|T|-2 \leq 4 \ell-6$ vertices of $V_{G}(T)$. Clearly, these vertices can have at most $4 \ell-6$ distinct colors. We consider all sets $C \subseteq\left\{1, \ldots, 2^{|T|}\right\}$ of distinct colors of size at most $4 \ell-6$ and for each $C$, we aim to find a minimum dominating set of $G$ whose vertices in $V_{G}(T)$ are colored by the maximum number of distinct colors and are colored exactly by the colors of $C$. Since we consider all possible choices of $C$, it holds for some $C$.

Toward this aim, we apply the following rule.

Rule 1 If there is an $x y$-vertex $w$ of $G$ for $x y \in E(T)$ and

1. $x, y \notin M_{u}$ for every $u \in V_{G}(T)$ with $c(u) \in C$; and

2. there is $v \in V_{G}(T)$ (with $c(v) \notin C$ ) such that $x, y \in M_{v}$,

then discard the current choice of $C$.

To see that the rule is safe, observe that if $D$ is minimum dominating set of $G$ whose vertices in $V_{G}(T)$ are colored exactly by the colors of $C$, then $w$ is dominated by some $x y$-vertex $w^{\prime}$. We have that $v \notin D$, because $c(v) \notin C$. Then it is straightforward to see that $D^{\prime}=\left(D \backslash\left\{w^{\prime}\right\}\right) \cup\{v\}$ is a minimum dominating set of $G$ whose vertices in $V_{G}(T)$ are colored by $|C|+1$ colors.

Now we are looking for a dominating set $D$ of minimum size such that $c\left(D \cap V_{G}(T)\right)=C$.

We use the following rule.

Rule 2 If there is a $Q$-vertex $u$ of $G$ for non-empty $Q \subseteq V(T)$ such that

1. $c(u) \notin C$; and

2. there is $c \in C$ such that for every $v \in V_{G}(T)$ with $c(v)=c, v$ dominates $u$,

then delete $u$.

To see that the rule is safe, observe that $u$ cannot be included in a dominating set $D$ of minimum size such that $c\left(D \cap V_{G}(T)\right)=C$ and $u$ is dominated by any set $D$ such that $c\left(D \cap V_{G}(T)\right)=C$.

Let

$A=\left\{x y \in E(T) \mid x, y \in M_{u}\right.$ for some $u \in V_{G}(T)$ such that $\left.c(u) \in C\right\}$

$A^{\prime}=\left\{x y \in E(T) \mid x, y \in M_{u}\right.$ for some $u \in V_{G}(T)$ such that $c(u) \notin C$ and

$x, y \notin M_{v}$ for $v \in V_{G}(T)$ such that $c(v) \in C$ \}. 
Observe that because of Rule 1, there are no $e$-vertices for $e \in A^{\prime}$. We contract the edges $e \in A \cup A^{\prime}$. Denote by $\hat{T}$ the tree obtained from $T$ tree and let $\hat{T}^{\prime}$ be the tree obtained from $T^{\prime}$ by contracting the paths that correspond to the contracted edge. We also construct the graph $\hat{G}$ that is obtained from $G$ by contracting these edges of $T$ and we also construct its $\hat{T}$-representation $\hat{\mathscr{M}}=\left\{\hat{M}_{v}\right\}_{v \in V(\hat{G})}$ where $\hat{M}_{v} \in V\left(\hat{T}^{\prime}\right)$ for $v \in V(\hat{G})$. We set $\hat{c}=\left.c\right|_{V(\hat{G})}$ and for every $x \in V(\hat{T})$ define

$$
C_{x}=\left\{c, \exists u \in V_{\hat{G}}(x) \text { s.t. } \hat{c}(u)=c\right\} .
$$

Observe that $\hat{\mathscr{M}}$ is a nice $\hat{T}$-representation of $\hat{G}$. Indeed, for every $x y \in E(T)$ such that $x, y \in M_{u}$ for $u \in V_{G}(T)$ we have that $x y \in A$ if $c(u) \in C$ and $x y \in A^{\prime}$ if $c(u) \notin C$ because of Rule 2, and all such edges $x y$ are contracted.

Combining Lemmas 5 and 6 we obtain that $D$ is a dominating set of minimum size with $C=c\left(D \cap V_{G}(T)\right)$ if and only if $D$ is a dominating set of $G^{\prime}$ of minimum size such that $C=\hat{c}\left(D \cap V_{\hat{G}}(\hat{T})\right)$. Note that the condition $C=\hat{c}\left(D \cap V_{\hat{G}}(\hat{T})\right)$ is equivalent to the condition that for every $x \in V(\hat{T}), C_{x}=\hat{c}\left(D \cap V_{\hat{G}}(x)\right)$, because the $\hat{T}$-representation of $\hat{G}$ is nice and $C_{x} \cap C_{y}=\emptyset$ for distinct $x, y \in V(\hat{T})$.

We set $d=|T|+\ell-1 \leq 3 \ell-3$ and apply the next rule.

Rule 3 If there is $x \in V(\hat{T})$ with $\left|C_{x}\right|>d$, then discard the current choice of $C$.

To see that the rule is safe, assume that the input graph $G$ has a minimum dominating set $D$ whose vertices in $V_{G}(T)$ are colored exactly by the colors of $C$. By Lemma 3, we have that if a set of nodes $X$ of $T$ is contracted into a single vertex $x$ of $\hat{T}$, then $D$ has at most $|X|+\ell-1$ vertices whose models contain a vertex of $X$ and, therefore, the number of vertices colored by the colors of $C_{x}$ in $D$ is at most $d$.

We arbitrarily select a node $r$ to be the root of $\hat{T}$ and $\hat{T}^{\prime}$ respectively. Then we apply Lemma 7 to the instance $\left(\hat{T}, k, d, \hat{c},\left\{C_{x}\right\}_{x \in V(\hat{T})}\right)$ of Dominating Set Extension.

Recall that Dominating Set Extension is a promise problem. If the algorithm from Lemma 7 returns a yes-answer, it means that there is a dominating set $D$ of $\hat{G}$ of size at most $k$ such that for each $x \in V(\hat{T}), C_{x}=c\left(D \cap V_{\hat{G}}(x)\right)$. This means that the input graph $G$ has a dominating set of size at most $k$. Still, if the promise is false, the algorithm can return an incorrect no-answer. Recall that the promise of DominATING Set Extension is the following: for every dominating set $D$ of $\hat{G}$ of minimum size with the properties that

(a) $D$ has at most $d x$-vertices for $x \in V(\hat{T})$,

(b) for each $x \in V(\hat{T}), C_{x} \subseteq c\left(D \cap V_{G}(\hat{x})\right)$,

it holds that the number of nodes $x \in V(\hat{T})$ such that $D$ contains an $x$-vertex is maximum and for each $x \in V(\hat{T}), C_{x}=c\left(D \cap V_{G}(\hat{x})\right)$. By Lemmas 5 and 6 , we have that if $C$ is chosen in such a way that $G$ has a minimum dominating set $D$ that has the maximum number of vertices of $V_{G}(T)$ and whose vertices in $V_{G}(T)$ are colored exactly by the colors of $C$, then this promise holds for the corresponding instance of Dominating Set Extension constructed for this choice of $C$. Therefore, if $(G, k)$ is a

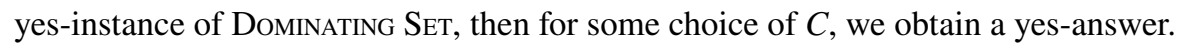


To evaluate the running time of the algorithm, observe that $T, T^{\prime}$ and the representation $\mathscr{M}$ are constructed in polynomial time by the algorithm of Habib and Stacho [20]. The coloring function $c: V_{G}(T) \rightarrow\left\{1, \ldots, 2^{|T|}\right\}$ can be constructed in time $2^{\mathscr{O}(\ell)} \cdot n^{\mathscr{O}(1)}$. Then we construct $2^{\mathscr{O}\left(\ell^{2}\right)}$ sets $C \subseteq\left\{1, \ldots, 2^{|T|}\right\}$ of size at most $6 \ell-8$ and it can be done in time $2^{\mathscr{O}\left(\ell^{2}\right)}$. For each $C$, the Rules 1 and 2 can be applied in polynomial time. Similarly, the construction of the instance $\left(\hat{T}, k, d, \hat{c},\left\{C_{x}\right\}_{x \in V(\hat{T})}\right)$ of Dominating Set Extension for a given $C$ can be done in polynomial time. Clearly, Rule 3 can be applied in polynomial time. Then we solve the constructed instance of Dominating SET Extension in time $2^{\mathscr{O}(\ell \log \ell)} \cdot n^{\mathscr{O}(1)}$. Hence, the total running time of the algorithm is $2^{\mathscr{O}\left(\ell^{2}\right)} \cdot n^{\mathscr{O}(1)}$.

The theorem immediately gives the following corollary for $T$-graphs.

Corollary 5 DominAting SET can be solved in time $2^{\mathscr{O}\left(|T|^{2}\right)} \cdot n^{\mathscr{O}(1)}$ for T-graphs if $T$ is a tree.

\subsection{A Polynomial Kernel for Clique}

It was observed in [10] that the CLIQUE problem is FPT for $H$-graphs when parameterized by the solution size $k$ and $\|H\|$ (even when no $H$-representation of $G$ is given). We show that CLIQUE admits a polynomial kernel when a representation is given.

Let $G$ be an $H$-graph with an $H$-representation $\mathscr{M}=\left\{M_{v}\right\}_{v \in V(G)}$ where, for the corresponding subdivision $H^{\prime}$ of $H$ and $v \in V(G), M_{v} \subseteq V\left(H^{\prime}\right)$. Recall that for $e \in E(H), v \in V(G)$ is an $e$-vertex if $M_{v}$ contains only subdivision nodes of $H^{\prime}$ from the path in $H^{\prime}$ corresponding to $e$ in $H$. We claim that we can find a maximum clique in $G$ that contains some $e$-vertex in polynomial time.

Lemma 8 Let $G$ be an $H$-graph given together with its H-representation. Then a clique of maximum size that contains at least one e-vertex for some e $\in E(G)$ can be found in time $\mathscr{O}\left(n^{3 / 2} m\right)$.

Proof Let $\mathscr{M}=\left\{M_{v}\right\}_{v \in V(G)}$ be an $H$-representation of $G$. For each $e$-vertex $u$ of $G$, we find a maximum clique $K$ such that $M_{u}$ is inclusion minimal for $K$, that is, there is no $v \in K$ with $M_{v} \subset M_{u}$. Let $e=x y$ for $x, y \in V(H)$ and denote by $P$ the $(x, y)$-path corresponding to $e$ in the subdivision $H^{\prime}$ of $G$. Since $u$ is an $e$-vertex, $M_{u} \subseteq V(P)$, that is, the nodes of $M_{u}$ form a subpath of $P$. Denote by $x^{\prime}$ and $y^{\prime}$ the end-vertices of the subpath. Note that it can happen that $x^{\prime}=y^{\prime}$. Because $M_{u}$ is an inclusion minimal model of a vertex of $K$, for every $v \in K, x^{\prime} \in M_{v}$ or $y^{\prime} \in M_{v}$. Consider $U=\left\{v \in V(G) \mid x^{\prime} \in M_{v}\right.$ or $\left.y^{\prime} \in M_{v}\right\}$. We have that finding $K$ in $G$ is equivalent to finding a maximum clique containing $u$ in $G^{\prime}=G[U]$.

Notice that $U$ can be partitioned into two cliques $K_{1}=\left\{v \in V(G) \mid x^{\prime} \in M_{v}\right\}$ and $K_{2}=\left\{v \in V(G) \mid y^{\prime} \in M_{v}\right.$ and $\left.x^{\prime} \notin M_{v}\right\}$. This means that $G^{\prime}$ is a cobipartite graph. A maximum clique in a cobipartite graph can be found in time $\mathscr{O}(\sqrt{n} m)$ by the algorithm of Hopcroft and Karp [21] as finding a maximum clique in $G^{\prime}$ is equivalent to finding a maximum independent set in the complement of $G^{\prime}$ that is a bipartite 
graph. Note that a maximum clique in $G^{\prime}$ always contains $u$, because $u$ is adjacent to every other vertex of $G^{\prime}$.

Since we consider all $e$-vertices to find a maximum clique containing some $e$-vertex for some $e \in E(H)$, the total running time is $\mathscr{O}\left(n^{3 / 2} m\right)$.

Now we a ready to construct our kernel.

Theorem 10 The CLIQUE problem for H-graphs admits a kernel with at most $(k-1)|H|$ vertices if an $H$-representation of the input graph is given, which can be computed in $\mathscr{O}\left(n^{3 / 2} m\right)$-time.

Proof Let $G$ be an $H$-graph with an $H$-representation $\mathscr{M}=\left\{M_{v}\right\}_{v \in V(G)}$ where $M_{v} \subseteq V\left(H^{\prime}\right)$ for the corresponding subdivision $H^{\prime}$ of $H$.

First, we use Lemma 8 to check whether $G$ has a clique of size at least $k$ that contains at least one $e$-vertex for some $e \in E(G)$. If we find such a clique we return a yes-answer. Assume that this is not the case. Let $G^{\prime}$ be the graph obtained from $G$ by the deletion of all $e$-vertices for $e \in E(H)$. We have that $G$ has a clique of size at least $k$ if and only if $G^{\prime}$ has a clique of size at least $k$.

If there is $x \in V(H)$ such that $V_{x}=\left\{v \in V\left(G^{\prime}\right) \mid x \in M_{v}\right\}$ has size at least $k$, then it is a clique of size at least $k$ and we return a yes-answer. Otherwise, we return $G^{\prime}$. Clearly, $\left|G^{\prime}\right| \leq(k-1)|H|$ in this case.

Acknowledgements Open Access funding provided by University of Bergen. We thank Saket Saurabh for bringing $H$-graphs to our attention. The first two authors have been supported by the Research Council of Norway via the projects "CLASSIS" and "MULTIVAL". The third author has been supported by the Polish National Science Centre grant PRELUDIUM DEC-2013/11/N/ST6/02706 and by the European Research Council (ERC) under the European Union's Horizon 2020 research and innovation programme, ERC consolidator grant DISTRUCT, agreement No 648527

Open Access This article is licensed under a Creative Commons Attribution 4.0 International License, which permits use, sharing, adaptation, distribution and reproduction in any medium or format, as long as you give appropriate credit to the original author(s) and the source, provide a link to the Creative Commons licence, and indicate if changes were made. The images or other third party material in this article are included in the article's Creative Commons licence, unless indicated otherwise in a credit line to the material. If material is not included in the article's Creative Commons licence and your intended use is not permitted by statutory regulation or exceeds the permitted use, you will need to obtain permission directly from the copyright holder. To view a copy of this licence, visit http://creativecommons.org/ licenses/by/4.0/.

\section{References}

1. Belmonte, R., Vatshelle, M.: On graph classes with logarithmic boolean-width. arXiv preprint, (2010) arXiv:1009.0216

2. Belmonte, R., Vatshelle, M.: Graph classes with structured neighborhoods and algorithmic applications. Theor. Comput. Sci. 511, 54-65 (2013) 
3. Berry, Anne, Bordat, Jean-Paul, Cogis, Olivier: Generating all the minimal separators of a graph. In: Graph-Theoretic Concepts in Computer Science: 25th International Workshop, WG'99 Ascona, Switzerland, June 17-19, pp. 167-172. Springer, Berlin (1999)

4. Biró, M., Hujter, M., Tuza, Z.: Precoloring extension. I. Interval graphs. Discret. Math. 100(1), 267$279(1992)$

5. Booth, K.S., Johnson, J.H.: Dominating sets in chordal graphs. SIAM J. Comput. 11(1), 191-199 (1982)

6. Bouchitté, V., Todinca, I.: Treewidth and minimum fill-in: grouping the minimal separators. SIAM J. Comput. 31(1), 212-232 (2001)

7. Bui-Xuan, B.-M., Telle, J.A., Vatshelle, M.: Boolean-width of graphs. Theor. Comput. Sci. 412(39), 5187-5204 (2011)

8. Bui-Xuan, B.-M., Telle, J.A., Vatshelle, M.: Fast dynamic programming for locally checkable vertex subset and vertex partitioning problems. Theor. Comput. Sci. 511, 66-76 (2013)

9. Chaplick, S., Töpfer, M., Voborník, J., Zeman, P.: On $H$-topological intersection graphs. In: International Workshop on Graph-Theoretic Concepts in Computer Science, pp. 167-179. Springer, Berlin (2017) arXiv:1608.02389

10. Chaplick, S., Zeman, P.: Combinatorial problems on $H$-graphs. In: The European Conference on Combinatorics, Graph Theory and Applications (EUROCOMB'17), Electronic Notes in Discrete Mathematics, vol. 61, pp. 223-229 (2017). arXiv:1706.00575

11. Cygan, M., Fomin, F.V., Kowalik, Ł., Lokshtanov, D., Marx, D., Pilipczuk, M., Pilipczuk, M., Saurabh, S.: Parameterized Algorithms. Springer, Berlin (2015)

12. Downey, R.G., Fellows, M.R.: Fundamentals of Parameterized Complexity. Texts in Computer Science. Springer, Berlin (2013)

13. Fellows, M.R., Hermelin, D., Rosamond, F.A., Vialette, S.: On the parameterized complexity of multiple-interval graph problems. Theor. Comput. Sci. 410(1), 53-61 (2009)

14. Fomin, F.V., Golovach, P.A., Raymond, J.-F.: On the tractability of optimization problems on $H$ -graphs. In: Azar Y., Bast H., Herman G. (eds.) 26th Annual European Symposium on Algorithms (ESA 2018), volume 112 of Leibniz International Proceedings in Informatics (LIPIcs), pp. 30:130:14, Dagstuhl, Germany, Schloss Dagstuhl-Leibniz-Zentrum fuer Informatik (2018)

15. Fomin, F.V., Lokshtanov, D., Saurabh, S., Zehavi, M.: Kernelization: Theory of Parameterized Preprocessing. Cambridge University Press, Cambridge (2019)

16. Fomin, F.V., Todinca, I., Villanger, Y.: Large induced subgraphs via triangulations and CMSO. SIAM J. Comput. 44(1), 54-87 (2015). arXiv:1309.1559

17. Gavril, F.: Algorithms for minimum coloring, maximum clique, minimum covering by cliques, and maximum independent set of a chordal graph. SIAM J. Comput. 1(2), 180-187 (1972)

18. Gavril, F.: The intersection graphs of subtrees in trees are exactly the chordal graphs. J. Combin. Theory Ser. B 16, 47-56 (1974)

19. Golumbic, M.C.: Algorithmic graph theory and perfect graphs. In: Annals of Discrete Mathematics, vol. 57. Elsevier Science B.V., Amsterdam, second edition, With a foreword by Claude Berge (2004)

20. Habib, M., Stacho, J.: Polynomial-time algorithm for the leafage of chordal graphs. In: AlgorithmsESA 2009, 17th Annual European Symposium, Copenhagen, Denmark, September 7-9, 2009. Proceedings, volume 5757 of Lecture Notes in Computer Science, pp. 290-300. Springer (2009)

21. Hopcroft, J.E., Karp, R.M.: An $\mathrm{n}^{5 / 2}$ algorithm for maximum matchings in bipartite graphs. SIAM J. Comput. 2(4), 225-231 (1973)

22. Jaffke, L., Kwon, O., Strømme, T.J.F., Telle, J.A.: Generalized distance domination problems and their complexity on graphs of bounded mim-width. In: Paul C., Pilipczuk M., (eds.) 13th International Symposium on Parameterized and Exact Computation (IPEC 2018), volume 115 of Leibniz International Proceedings in Informatics (LIPIcs), pp. 6:1-6:14, Dagstuhl, Germany, Schloss Dagstuhl-Leibniz-Zentrum fuer Informatik (2019)

23. Jaffke, L., Kwon, O., Telle, J.A.: Polynomial-time algorithms for the longest induced path and induced disjoint paths problems on graphs of bounded mim-width. In: Lokshtanov D., Nishimura N., (eds.) 12th International Symposium on Parameterized and Exact Computation (IPEC 2017), volume 89 of Leibniz International Proceedings in Informatics (LIPIcs), pp. 21:1-21:13, Dagstuhl, Germany, Schloss Dagstuhl-Leibniz-Zentrum fuer Informatik (2018)

24. Jaffke, L., Kwon, O., Telle, J.A.: A note on the complexity of feedback vertex set parameterized by mim-width. arXiv preprint, (November 2017). arXiv:1711.05157 
25. Kloks, T., Bodlaender, H., Müller, H., Kratsch, D.: Computing treewidth and minimum fill-in: all you need are the minimal separators. Algorithms-ESA'93, pp. 260-271 (1993)

26. Lin, I.-J., McKee, T., West, D.: The leafage of a chordal graph. Discuss. Math. Graph Theory 18(1), 23-48 (1998)

27. Pietrzak, K.: On the parameterized complexity of the fixed alphabet shortest common supersequence and longest common subsequence problems. J. Comput. Syst. Sci. 67(4), 757-771 (2003)

28. Raman, V., Saurabh, S.: Short cycles make W[1]-hard problems hard: FPT algorithms for W[1]hard problems in graphs with no short cycles. Algorithmica 52(2), 203-225 (2008)

Publisher's Note Springer Nature remains neutral with regard to jurisdictional claims in published maps and institutional affiliations.

\section{Affiliations}

\section{Fedor V. Fomin ${ }^{1}$. Petr A. Golovach ${ }^{1}$. Jean-Florent Raymond ${ }^{2} \mathbb{D}$}

Petr A. Golovach

petr.golovach@ii.uib.no

Jean-Florent Raymond

j-florent.raymond@uca.fr

1 Department of Informatics, University of Bergen, Bergen, Norway

2 CNRS, LIMOS, Université Clermont Auvergne, Clermont-Ferrand, France 